\title{
Stochastic suspensions of heavy particles
}

\author{
Jérémie Bec, ${ }^{1, \text { - }}$ Massimo Cencini, ${ }^{2,3}$ Rafaela Hillerbrand ${ }^{4}$ and Konstantin Turitsyn ${ }^{5,6}$ \\ ${ }^{1}$ Laboratoire Cassiopée, Observatoire de la Côte d'Azur, CNRS, \\ Université de Nice Sophia-Antipolis, Bd. de l'Observatoire, 06300 Nice, France \\ ${ }^{2}$ SMC INFM-CNR c/o Dip. di Fisica Università di Roma "La Sapienza", Piazzale A. Moro 2, 00185 Roma, Italy \\ ${ }^{3}$ CNR, Istituto dei Sistemi Complessi, Via dei Taurini 19, 00185 Roma, Italy \\ ${ }^{4}$ The Future of Humanity Institute, University of Oxford, Suite 8, \\ Littlegate House 16/17, StEbbe's Street, Oxford, OX11PT, United Kingdom \\ ${ }^{5}$ James Franck Institute, University of Chicago, Chicago, IL 60637, USA \\ ${ }^{6}$ Landau Institute for Theoretical Physics, Moscow, Kosygina 2, 119334, Russia
}

\begin{abstract}
Turbulent suspensions of heavy particles in incompressible flows have gained much attention in recent years. A large amount of work focused on the impact that the inertia and the dissipative dynamics of the particles have on their dynamical and statistical properties. Substantial progress followed from the study of suspensions in model flows which, although much simpler, reproduce most of the important mechanisms observed in real turbulence. This paper presents recent developments made on the relative motion of a pair of particles suspended in time-uncorrelated and spatially self-similar Gaussian flows. This review is complemented by new results. By introducing a time-dependent Stokes number, it is demonstrated that inertial particle relative dispersion recovers asymptotically Richardson's diffusion associated to simple tracers. A perturbative (homogeneization) technique is used in the small-Stokes-number asymptotics and leads to interpreting first-order corrections to tracer dynamics in terms of an effective drift. This expansion implies that the correlation dimension deficit behaves linearly as a function of the Stokes number. The validity and the accuracy of this prediction is confirmed by numerical simulations.
\end{abstract}

PACS numbers: 47.27.-i, 47.51.+a, 47.55.-t

Keywords: Stochastic flows; Inertial particles; Kraichnan model; Lyapunov exponent

\section{INTRODUCTION}

The current understanding of passive turbulent transport profited significantly from studies of the advection by random fields. In particular, flows belonging to the so-called Kraichnan ensemble - i. e. spatially self-similar Gaussian velocity fields with no time correlation — which was first introduced in the late 1960's by R.H. Kraichnan [1], led in the mid 1990's to a first analytical description of anomalous scaling in turbulence (see [2] for a review). More recently, much work is devoted to a generalization of this passive advection to heavy particles that, conversely to tracers, do not follow the flow exactly but lag behind it due to their inertia. The particle dynamics is thus dissipative even if the carrier flow is incompressible. This paper provides an overview of several recent results on the dynamics of very heavy particles suspended in random flows belonging to the Kraichnan ensemble.

The recent shift of focus to the transport of heavy particles is motivated by the fact that in many natural and industrial flows finite-size and mass effects of the suspended particles cannot be neglected. Important applications encompass rain formation [3, 4, 5] and suspensions of biological organisms in the ocean [6, 7, 8]. For practical purposes, the formation of particle clusters due to inertia is of central importance as the presence of such inhomogeneities significantly enhances interactions between the

*Electronic address: jeremie.bec@oca.eu suspended particles. However, detailed and reliable predictions on collision or reaction rates, which are crucial to many applications, are still missing.

Two mechanisms compete in the formation of clusters. First, particles much denser than the fluid are ejected from the eddies of the carrier flow and concentrate in the strain-dominated regions [9]. Second, the dissipative dynamics leads the particle trajectories to converge onto a fractal, dynamically evolving attractor [10, 11]. In many studies, a carrier velocity field with no time correlation and thus no persistent structures - is used to isolate the latter effect. As interactions between three or more particles are usually sub-dominant, most of the interesting features of mono-disperse suspensions can be captured by focusing on the relative motion of two particles separated by $\boldsymbol{R}$ :

$$
\ddot{\boldsymbol{R}}=-\frac{1}{\tau}[\dot{\boldsymbol{R}}-\delta \boldsymbol{u}(\boldsymbol{R}, t)],
$$

where dots denote time derivatives and $\tau$ the particle response time. The fluid velocity difference $\delta \boldsymbol{u}$ is a Gaussian vector field with correlation

$$
\left\langle\delta u^{i}(\boldsymbol{r}, t) \delta u^{j}\left(\boldsymbol{r}^{\prime}, t^{\prime}\right)\right\rangle=2 b^{i j}\left(\boldsymbol{r}-\boldsymbol{r}^{\prime}\right) \delta\left(t-t^{\prime}\right) .
$$

In order to model turbulent flows, the tensorial structure of the spatial correlation $b^{i j}(\boldsymbol{r})$ is chosen to ensure incompressibility, isotropy and scale invariance, namely

$$
b^{i j}(\boldsymbol{r})=D_{1} r^{2 h}\left[(d-1+2 h) \delta^{i j}-2 h r^{i} r^{j} / r^{2}\right],
$$

where $h$ relates to the Hölder exponent of the fluid velocity field and $D_{1}$ measures the intensity of its fluctuations. 
In particular, $h=1$ corresponds to a spatially differentiable velocity field, mimicking the dissipative range of a turbulent flow, while $h<1$ models rough flows, as in the inertial range of turbulence. In this paper we mostly focus on space dimensions $d=1$ and $d=2$; extensions to higher dimensions are just sketched.

The above depicted model flow has the advantage that the particle dynamics is a Markov process. In particular, Gaussianity and $\delta$-correlation in time of the fluid velocity field imply that the probability density $p\left(\boldsymbol{r}, \boldsymbol{v}, t \mid \boldsymbol{r}_{0}, \boldsymbol{v}_{0}, t_{0}\right)$ of finding the particles at separation $\boldsymbol{R}(t)=\boldsymbol{r}$ and with relative velocity $\dot{\boldsymbol{R}}(t)=\boldsymbol{v}$ at time $t$, when $\boldsymbol{R}\left(t_{0}\right)=\boldsymbol{r}_{0}$ and $\dot{\boldsymbol{R}}\left(t_{0}\right)=\boldsymbol{v}_{0}$ is a solution of the Fokker-Planck equation

$$
\partial_{t} p+\sum_{i}\left(\partial_{r}^{i}-\frac{1}{\tau} \partial_{v}^{i}\right)\left(v^{i} p\right)-\sum_{i, j} \frac{b^{i j}(\boldsymbol{r})}{\tau^{2}} \partial_{v}^{i} \partial_{v}^{j} p=0
$$

with the initial condition $p\left(\boldsymbol{r}, \boldsymbol{v}, t_{0}\right)=\delta\left(\boldsymbol{r}-\boldsymbol{r}_{0}\right) \delta\left(\boldsymbol{v}-\boldsymbol{v}_{0}\right)$. To maintain a statistical steady state, the Fokker-Planck equation (4) as well as the stochastic differential equation (11) should be supplemented by boundary conditions, here chosen to be reflective at a given distance $L$.

For smooth flows $(h=1)$, the intensity of inertia is generally measured by the Stokes number St, defined as the ratio between the particle response time $\tau$ and the fluid characteristic time scale. For St $\rightarrow 0$, particles recover the incompressible dynamics of tracers. In the opposite limit where St is very large, inertia effects dominate and the dynamics approaches that of free particles. In the above depicted model, the Stokes number is defined by nondimensionalizing $\tau$ by the typical fluid velocity gradient, i.e. $\mathrm{St}=D_{1} \tau$. Note that by rescaling the physical time by $\tau$, it is straightforward to recognize that the dynamics depends solely on St.

Similarly it can be checked that in rough flows $(h<1)$ - with an additional rescaling of the distances by a factor $\left(D_{1} \tau\right)^{1 /(2-2 h)}$ - the dynamics of a particle pair at a distance $r$ only depends on the local Stokes number $\operatorname{St}(r)=D_{1} \tau / r^{2(1-h)}$. This dimensionless quantity, first introduced in [12] and later used in [13], is a generalization of the Stokes number to cases in which the fluid turnover times depend on the observation scale. At large scales, $\operatorname{St}(r) \rightarrow 0$ and inertia becomes negligible. Particle dynamics thus approaches that of tracers. At small scales, $\operatorname{St}(r) \rightarrow \infty$ and the particle and fluid motions decorrelate, so that the inertial particles move ballistically. In both the large and small Stokes number asymptotics, particles distribute uniformly in space, while inhomogeneities are expected at intermediate values of $\mathrm{St}(r)$.

The paper is organized as follows. In Section III, an approach originally proposed in [14] is used to reduce the dynamics of the particle separation to a system of three stochastic equations with additive noises. This formulation is useful for both numerical and analytical purposes, particularly when studying the statistical properties of particle pairs. In Section III, we introduce the correlation dimension to quantify clustering as well as the approaching rate which measures collisions. Numerical results for these quantities are reported. In Section IV we introduce the notion of time-dependent Stokes number which makes particularly transparent the interpretation of the behavior of the long-time separation between particles. We show how Richardson dispersion, as for tracers, is recovered in the long time asymptotics. Section $\mathbf{V}$ briefly summarizes some exact results that can be obtained for the one-dimensional case. Sections [VI and VII are dedicated to the small and large Stokes number asymptotics, respectively. In particular, the former one presents an original perturbative approach which turned out to predict, in agreement with numerical computations, the behavior of the correlation dimension that characterizes particle clusters. Finally, Section VIII encompasses conclusions, open questions and discusses the relevance of the considered model for real suspensions in turbulent flows.

\section{REDUCED DYNAMICS FOR THE TWO-POINT MOTION}

In this Section we focus on planar suspensions $(d=2)$. Following the approach proposed in 14] and with the notation $R=|\boldsymbol{R}|$, the change of variables

$$
\begin{aligned}
\sigma_{1} & =(L / R)^{1+h} \boldsymbol{R} \cdot \dot{\boldsymbol{R}} / L^{2}, \\
\sigma_{2} & =(L / R)^{1+h}|\boldsymbol{R} \wedge \dot{\boldsymbol{R}}| / L^{2}, \\
\rho & =(R / L)^{1-h}
\end{aligned}
$$

is introduced to reduce the original system of $2 d=4$ stochastic equations to the following one of only three equations

$$
\begin{aligned}
& \dot{\sigma}_{1}=-\sigma_{1} / \tau-\left[h \sigma_{1}^{2}-\sigma_{2}^{2}\right] / \rho+\sqrt{C} \eta_{1} \\
& \dot{\sigma}_{2}=-\sigma_{2} / \tau-(1+h) \sigma_{1} \sigma_{2} / \rho+\sqrt{(1+2 h) C} \eta_{2} \\
& \dot{\rho}=(1-h) \sigma_{1}
\end{aligned}
$$

where $C=2 D_{1} /\left(\tau L^{1-h}\right)^{2}$ and $\eta_{i}$ denote two independent standard white noises. Reflective boundary conditions at $R=L$ in physical space imply reflection at $\rho=1$. Note that $\sigma_{1}$ and $\sigma_{2}$ are proportional to the longitudinal and to the transversal relative velocities between the two particles. In the smooth case $(h=1)$, one has $\rho=1$ and equations (8) and (9) decouple from (10). The particle separation $R$ then evolves as

$$
\dot{R}=\sigma_{1}(t) R .
$$

Besides this simple evolution and the reduction of the number of variables from $2 d$ to only three, the change of variables $\{\boldsymbol{R}, \dot{\boldsymbol{R}}\} \mapsto\left\{\rho, \sigma_{1}, \sigma_{2}\right\}$ has several other advantages. For instance the noise, which is multiplicative in the original dynamics (1), becomes additive in the reduced system (8) - (10). However, this simplification is counter-balanced by the presence of nonlinear drift terms. Note that in dimensions higher than two, there is an additional term $\propto 1 / \sigma_{2}$, which is due to the Itô formula [15, 16]. 

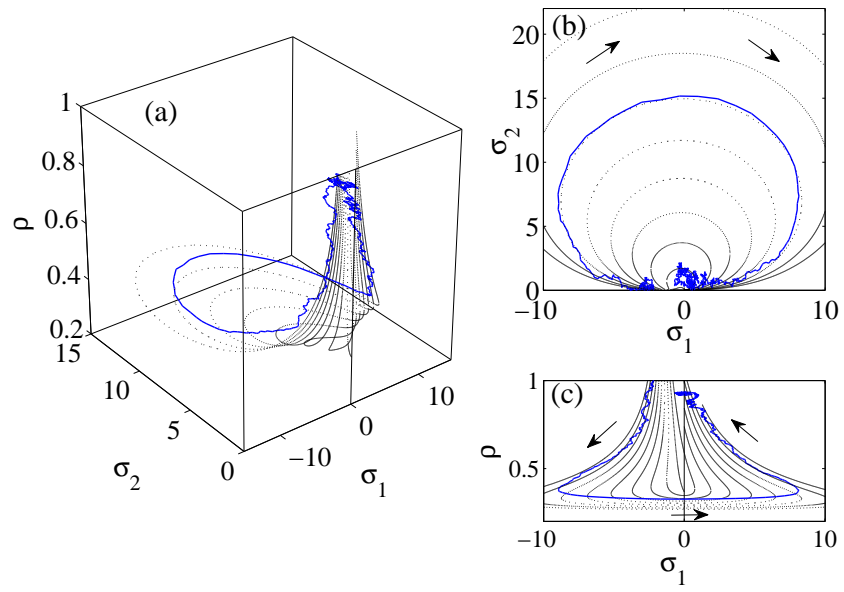

FIG. 1: Sketch of the reduced dynamics (8) - (10) for $h=0.7$. The dotted lines represent the drift. The solid line depicts a random trajectory with $\operatorname{St}(L)=1$. (a) full $\left(\sigma_{1}, \sigma_{2}, \rho\right)$-space, (b) projection on $\rho=0$ plane, and (c) on the $\sigma_{2}=0$ plane.

Figure 1 sketches the deterministic drift and shows a typical trajectory in the reduced space. This dynamics can be qualitatively described as follows. The line $\sigma_{1}=\sigma_{2}=0$ acts as a stable fixed line for the drift. Hence a typical trajectory spends a long time diffusing around it, until the noise realization becomes strong enough to let the trajectory escape from the vicinity of this line. Whenever this happens with a positive longitudinal relative velocity $\left(\sigma_{1}>0\right)$, the trajectory is pulled back to the stable line by the quadratic terms in the drift. Conversely, if $\sigma_{1}<0$ and $h \sigma_{1}^{2}+\sigma_{1} \rho-\sigma_{2}^{2}<0$, the drift pushes the trajectory towards larger negative values of $\sigma_{1}$. Then the particles get closer to each other and $\rho$ decreases, until the quadratic terms in equations (8) and (9) become dominant. The trajectory then loops back in the $\left(\sigma_{1}, \sigma_{2}\right)$-plane, approaching the stable line from its right. It is during these loops that the inter-particle distance $R$ becomes substantially small. The loops thus provide the main mechanisms for cluster formation.

\section{Velocity statistics}

Numerical simulations show that the probability density function (pdf) of the longitudinal relative velocity $\sigma_{1}$ displays algebraic tails at large positive and negative values (see Fig. 2). As will become clear in the sequel, these power-law tails are a signature of the above-mentioned large loops. Let us consider the cumulative probability $P^{<}(\sigma)=\operatorname{Pr}\left(\sigma_{1}<\sigma\right)$ for $\sigma \ll-1$. This quantity can be estimated as the product of (i) the probability to start a sufficiently large loop in the $\left(\sigma_{1}, \sigma_{2}\right)$-plane that reaches values smaller than $\sigma$ and (ii) the fraction of time spent by the trajectory at $\sigma_{1}<\sigma$. Within a distance of the order of unity from the line $\sigma_{1}=\sigma_{2}=0$, the quadratic terms in the drift are subdominant and can be disregarded. Then $\sigma_{1}$ and $\sigma_{2}$ can be approximated by two

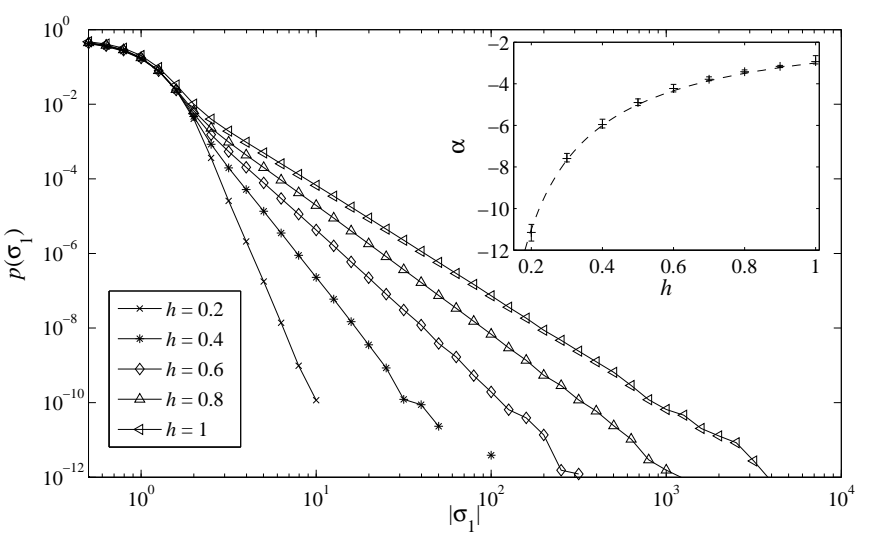

FIG. 2: Log-log plot of the pdf of $\sigma_{1}$ for $\operatorname{St}(L)=1$ for five values of the fluid Hölder exponent $h$. Power-law tails are always observed, $p(\sigma) \propto|\sigma|^{-\alpha}$. Inset: exponent $\alpha$ versus $h$; the dashed line is the theoretical prediction $\alpha=1+2 / h$.

independent Ornstein-Uhlenbeck processes. Conversely, at sufficiently large distances from that line, only the quadratic terms in the drift contribute and the noise is negligible.

Within this simplified dynamics, a loop is initiated at a time $t_{0}$ for which $\sigma_{1}\left(t_{0}\right)<-1$ and $\sigma_{2}\left(t_{0}\right) \ll\left|\sigma_{1}\left(t_{0}\right)\right|$. Once these conditions are fulfilled, the trajectory performs a loop in the $\left(\sigma_{1}, \sigma_{2}\right)$-plane and both $\left|\sigma_{1}(t)\right|$ and $\sigma_{2}(t)$ become very large. The maximum distance from the stable line, which gives an estimate of the loop radius, is reached when $\sigma_{2}$ is of the order of $\left|\sigma_{1}\right|$. Let $t^{*}$ denote the time when this happens, i.e. $\sigma_{2}\left(t^{*}\right) /\left|\sigma_{1}\left(t^{*}\right)\right|=\mathrm{O}(1)$. When neglecting the noise, this condition leads to the following estimate for the loop radius

$$
\left|\sigma_{1}\left(t^{*}\right)\right| \propto\left[\sigma_{1}\left(t_{0}\right)+\rho\left(t_{0}\right) / \tau\right]\left|\tau \sigma_{1}\left(t_{0}\right)\right|^{h}\left(\tau \sigma_{2}\left(t_{0}\right)\right)^{-h},
$$

see [13] for details. In order to reach velocity differences such that $\sigma_{1}<\sigma \ll-1$, the radius of the loop has to be larger than $|\sigma|$. From (12) this implies that $\sigma_{2}\left(t_{0}\right)$ has to be smaller than $|\sigma|^{-1 / h}$. In order to evaluate contribution (i), one has to estimate the probability to have $\sigma_{1}\left(t_{0}\right) \lesssim-1$ and $\sigma_{2}\left(t_{0}\right)<|\sigma|^{-1 / h}$ from the dynamics in the vicinity of the origin. Approximating the two velocity differences $\sigma_{1}$ and $\sigma_{2}$ by independent OrnsteinUhlenbeck processes close to the line $\sigma_{1}=\sigma_{2}=0$, the first condition gives an order-unity contribution, while the second has a probability $\propto|\sigma|^{-1 / h}$. For estimating (ii), we neglect the noise in the dynamics far from the stable line. The probability is then given by the fraction of time spent at $\sigma_{1}<\sigma$ which is proportional to $\sigma_{2}\left(t_{0}\right) \propto|\sigma|^{-1 / h}$. Put together, the two contributions yield $P^{<}(x) \propto|\sigma|^{-2 / h}$ when $\sigma \ll-1$. Thus the negative tail of the pdf of $\sigma_{1}$ behaves as $\propto|\sigma|^{-\alpha}$, with $\alpha=1+2 / h$.

During the large loops, the trajectories equally reach large positive values of $\sigma_{1}$ and of $\sigma_{2}$. Again the fraction of time spent at both $\sigma_{1}$ and $\sigma_{2}$ larger than $\sigma \gg 1$ can be estimated as $\sigma^{-1 / h}$. Hence, the pdf of both longitudinal $\sigma_{1}$ and transversal $\sigma_{2}$ velocity differences have algebraic 
left and right tails with exponent $\alpha$. Both tails are depicted in Fig. 2], where the inset shows that the numerical measurements are in good agreement with the predicted value of $\alpha$. The relation between $\alpha$ and the Hölder exponent $h$ implies in particular that $\alpha=3$ in the smooth case, while it increases with decreasing $h$. Moreover, it follows straightforwardly from (8) - (10) that during the loops $\rho(t) \propto \rho\left(t_{0}\right)^{h}$ when $\rho\left(t_{0}\right) \ll 1$. Hence it becomes less and less probable to reach smaller values of $\rho$ as $h$ decreases. In other words, particle clustering should be very strong for smooth flows and becomes weaker when the flow roughness is increased. This prediction is confirmed by the numerical studies presented in next Section.

Finally it should be pointed out that although the change of variables (5) - (7) can be applied equally in three dimensions, the above analysis does not carry over to higher dimensions. Firstly, as already pointed out, an additional drift term arises. This Itô-term renders a straightforward derivation of an analytic solution for the deterministic drift impossible. Secondly, for higher dimensions the fixed point of the reduced dynamics is located far from the origin, see [16]. Hence the approximations made above for $d=2$ are not applicable. Careful numerical studies are needed to understand whether or not algebraic tails are also present in higher dimensions.

\section{CORRELATION DIMENSION AND APPROACHING RATE}

Particle clustering is often quantified by the radial distribution function $g(r)$, which is defined as the ratio between the number of particles inside a thin shell of radius $r$ centered on a given particle and the number which would be in this shell if the particles were uniformly distributed. This quantity enters models for the collision kernel [17]. Following [10, 13, 16, 18], we consider a different, but related way to characterize particle clustering. Instead of the radial distribution function we evaluate the correlation dimension $\mathcal{D}_{2}$ of the set formed by the particles. This dimension is widely used in dissipative dynamical system theory and in fractal geometry (see, e.g., [19, 20]). It is defined as the exponent of the powerlaw behavior at small scales of the probability $P_{2}(r)$ of finding two particles at a distance $R<r$ :

$$
\mathcal{D}_{2}=\lim _{r \rightarrow 0} d_{2}(r), \quad d_{2}(r)=\frac{\mathrm{d} \ln P_{2}(r)}{\mathrm{d} \ln r},
$$

where the logarithmic derivative $d_{2}(r)$ is called the local correlation dimension. $\mathcal{D}_{2}$ relates to the radial distribution function via $\ln g(r) / \ln r \rightarrow \mathcal{D}_{2}-d$ for $r \rightarrow 0$. For uniformly distributed particles, $\mathcal{D}_{2}=d$, so that $g(r)=\mathrm{O}(1)$. On the contrary, when particles cluster on a fractal set, $\mathcal{D}_{2}<d$ and $g(r)$ diverges for $r \rightarrow 0$. This was also found numerically in [17].

Depending on whether the carrier flow is spatially smooth $(h=1)$ or rough $(h<1), \mathcal{D}_{2}$ and $d_{2}(r)$ behave differently. In the former case, random dynamical system theory 21] suggests that within the $2 \times d$ positionvelocity phase space, particles converge onto a multifractal set with correlation dimension $0<\overline{\mathcal{D}}_{2}<2 d$. Here $\overline{\mathcal{D}}_{2}$ denotes the correlation dimension in the full phase space. It is defined in complete analogy to $\mathcal{D}_{2}$ through the scaling behavior of the probability $\bar{P}_{2}(r)$ to find two particles at a distance less than $r$ in phase space:

$$
\bar{P}_{2}(r) \sim r^{\overline{\mathcal{D}}_{2}} \quad \text { for } \quad r \rightarrow 0 .
$$

The distance $r$ is now computed by using the phase-space Euclidean norm $\sqrt{|\boldsymbol{R}|^{2}+\left|\boldsymbol{V} / D_{1}\right|^{2}} ; \boldsymbol{V}$ is normalized by the typical fluid velocity gradient $D_{1}$ for dimensional reasons. The physical-space correlation dimension $\mathcal{D}_{2}$ is actually the dimension of the projection of the set from the full phase space onto the position space, and it is also expected to be fractal (see Section VII for details on the relation between $\overline{\mathcal{D}}_{2}$ and $\mathcal{D}_{2}$ ). We focus in this Section on quantifying clustering in position space and hence consider only $\mathcal{D}_{2}$ and $d_{2}(r)$.

Balkovsky et al. argued in [43] that particles do not form fractal sets in non-smooth flows because the correlation function of the particle density field should be a stretched exponential. Clustering and inhomogeneities are hence not quantified by a fractal dimension but by the detailed scale dependence of $d_{2}(r)$. However, as discussed in the Introduction, one expects the statistical properties of two particles separated by a distance $r$ in a flow with Hölder exponent $h$ to depend on the local Stokes number $\operatorname{St}(r)=D_{1} \tau / r^{2(1-h)}$ only, which for smooth flows degenerates to a scale independent number, $\operatorname{St}(r)=\mathrm{St}=D_{1} \tau$. In rough flows, at scales small enough, particles move ballistically and distribute homogeneously as the Lagrangian motion is too fast for the particles to follow $(\operatorname{St}(r) \rightarrow \infty$ as $r \rightarrow 0$ ) and hence $\mathcal{D}_{2}=d$ for all particle response times $\tau$. However, information on the inhomogeneities of the particle distribution at larger scales can still be obtained through the scale-dependence of the local correlation dimension $d_{2}(r)$ defined in (13).

The relevance of the local Stokes number and of the local correlation dimension is confirmed by numerical experiments of planar suspensions. Simulations were performed by directly integrating the reduced system described in previous Section. Figure 3 shows $d_{2}(r)$ as a function of $\operatorname{St}(r)$ for various values of $h$. The curves obtained with different values of the response time $\tau$ collapse onto the same $h$-dependent master curve once the scale dependency is reabsorbed by using $\operatorname{St}(r)$. In the plot, only scales far from the boundaries were considered, as otherwise the self-similarity of the fluid flow is broken. The data for $h=1$ estimate the limit of $d_{2}(r)$ as $r \rightarrow 0$, and so correspond to the value of the correlation dimension $\mathcal{D}_{2}$. As anticipated in the previous Section, Fig. 3 also shows that clustering is weakening when the roughness of the fluid velocity increases (i.e. when $h$ decreases). In particular, $\min _{r}\left\{d_{2}(r)\right\}$ gets closer to $d$, i.e. particles approach the uniform distribution as $h \rightarrow 0$. Finally notice that for $\mathrm{St}(r) \rightarrow 0$, i.e. at large scales in rough flows, 
$d_{2}(r) \rightarrow d$ as well. This is due to the fact that at these scales the Lagrangian motion becomes much slower than the relaxation time of the particles. The particles thus recover the tracer limit and distribute homogeneously. As we will see in SectionVI the local dimension $d_{2}(r)$ tends linearly to the space dimension $d$ when $\operatorname{St}(r) \rightarrow 0$ with a factor whose dependence on $h$ and $d$ can be obtained analytically by perturbative methods.

The radial distribution function and hence the correlation dimension give only partial information on the rate at which particles collide. Indeed, in order to evaluate the collision rate, one needs to know not only the probability that the particles are close to each other, but also their typical velocity difference. Here, following [18], we study the approaching rate $\kappa(r)$ defined as the flux of particles that are separated by a distance less than $r$ and approach each other, i.e.

$$
\kappa(r)=\langle\dot{\boldsymbol{R}} \cdot \boldsymbol{R} /|\boldsymbol{R}| \Theta(-\dot{\boldsymbol{R}} \cdot \boldsymbol{R} /|\boldsymbol{R}|) \Theta(r-|\boldsymbol{R}|)\rangle,
$$

where $\Theta$ denotes the Heaviside function and the average is defined on the Lagrangian trajectories. As detailed in [18], $\kappa(r)$ is related to the binary collision rate in the framework of the so-called ghost collision scheme 23]. Within this approach collision events are counted while allowing particles to overlap instead of scattering. At small separations, $\kappa(r)$ behaves as a power law. This algebraic behavior allows defining a local Hölder exponent $\gamma(r)$ for the particle velocities

$$
\gamma(r)=\frac{\ln \kappa(r)}{\ln r}-d_{2}(r) .
$$

In the above definition the contribution from clustering, accounted for by the local correlation dimension $d_{2}(r)$, is removed. The local Hölder exponent $\gamma(r)$, similarly to $d_{2}(r)$, tends to a finite limit $\Gamma$ as $r \rightarrow 0$ which, for

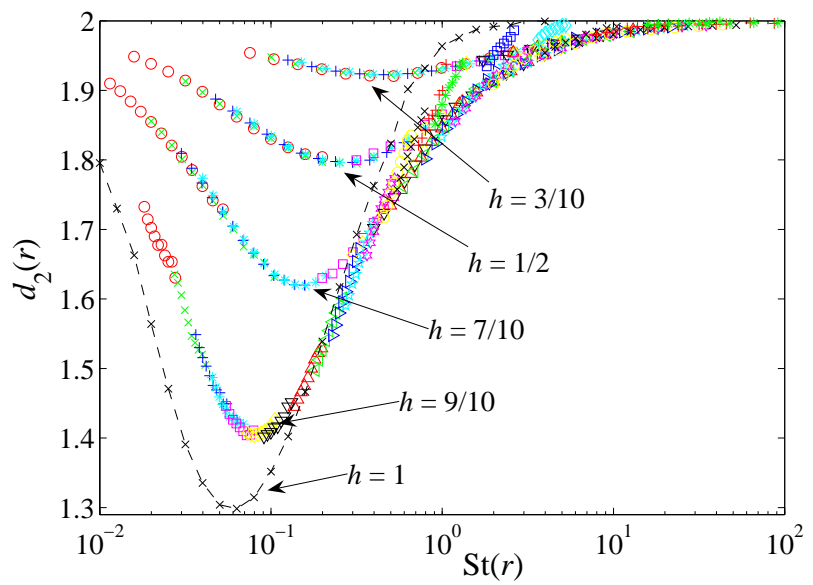

FIG. 3: Local correlation dimension $d_{2}(r)$ versus the scaledependent Stokes number $\operatorname{St}(r)=D_{1} \tau / r^{2(1-h)}$ for twodimensional flows with different $h$. Symbols denote different particle response times $\tau$. For $h=1, \mathcal{D}_{2}=d_{2}(r \rightarrow 0)$ is displayed and $\operatorname{St}(r)=\mathrm{St}=D_{1} \tau$. particles suspended in a smooth flow $(h=1)$, depends non-trivially on the Stokes number.

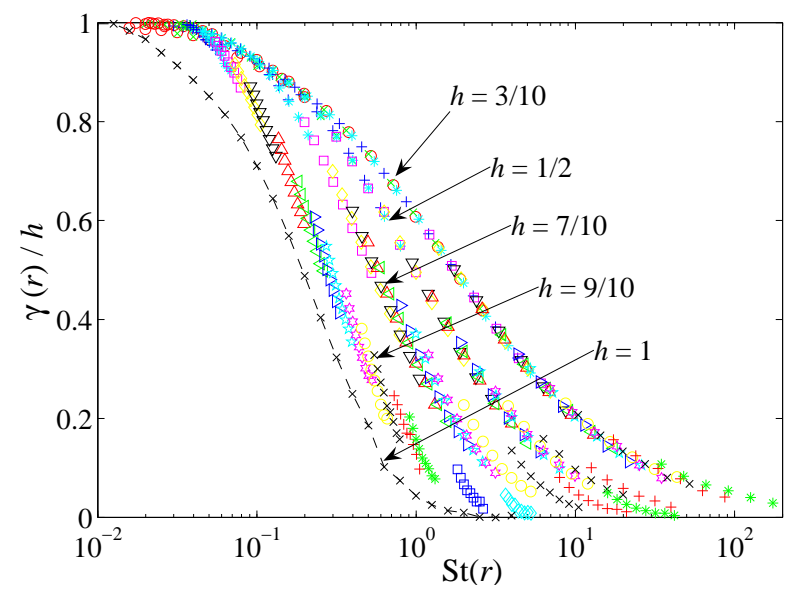

FIG. 4: Ratio between the local Hölder exponent $\gamma(r)$ of the particle velocity and that of the fluid $h$ versus $\operatorname{St}(r)$. The symbols in each curve refer to different values of the particle response time $\tau$. As in Fig. 3] for $h=1$, the small scale limiting value $\Gamma$ is depicted.

Figure 4 shows numerical estimations of $\gamma(r) / h$ as a function of $\operatorname{St}(r)$ for various values of $h$. In the smooth case $(h=1)$, the limit value $\Gamma$ decreases from $\Gamma=1$ for $\mathrm{St}=0$, which corresponds to a differentiable particle velocity field, to $\Gamma=0$ for $\mathrm{St} \rightarrow \infty$, which means that particles move with uncorrelated velocities [16]. The fact that $\Gamma<1$ is due to the contribution of caustics appearing in the particle velocity field [15, 18, 24, 25, 26] (see Sect. V for a discussion in $d=1$ ). Similarly, in non-smooth flows $\gamma(r)$ is asymptotically equal to the fluid Hölder exponent $h$ at large scales $(\mathrm{St}(r) \rightarrow 0)$, and approaches 0 at very small scales $(\operatorname{St}(r) \rightarrow \infty)$. Therefore, all the relevant information is entailed in the intermediate behavior of $\gamma(r)$. The latter should only depend on the fluid Hölder exponent and on the local Stokes number, as confirmed by the collapse observed in Fig. 4. Note that the transition from $\gamma(r)=h$ to $\gamma(r)=0$ shifts towards larger values of the local Stokes number and broadens as $h$ decreases. The fact that $\gamma(r)=h$ for $r \rightarrow \infty$ implies that the particles should asymptotically experience Richardson diffusion just as tracers (see Sect. IV for details). For comments on how the findings reported in this Section translate to realistic turbulent flows, we refer the reader to SectionVIII.

\section{STRETCHING RATE AND RELATIVE DISPERSION}

This Section is devoted to the study of the behavior of the distance $R(t)$ between two particles at intermediate times $t$ such that $R(0) \ll R(t) \ll L$. For convenience, we drop the reflective boundary condition at $R=L$ and consider particles evolving in an unbounded domain. 


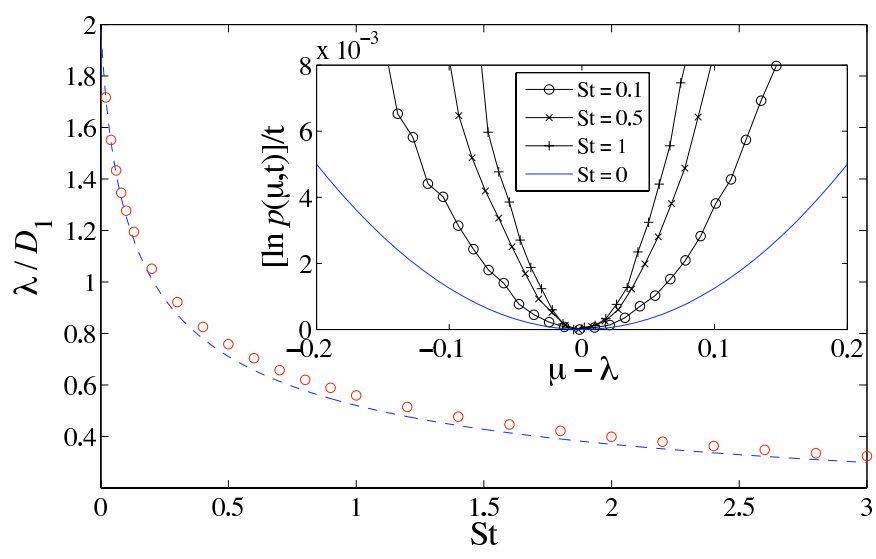

FIG. 5: Lyapunov exponent versus St: the circles are the numerical measurements while the dashed line corresponds to Eq. (20). Inset: rate function $H$ associated to the large deviations of the stretching rate $\mu$ for three values of St; the solid line corresponds to $H$ for tracers for, whose analytic expression is known (see, e.g., [2]).

We first consider a differentiable fluid velocity field $(h=1)$. In this case, the time evolution of the distance $R(t)$ is given by (11), so that

$$
R(t)=R(0) \exp \left[\int_{0}^{t} \sigma_{1}\left(t^{\prime}\right) \mathrm{d} t^{\prime}\right]
$$

and the particle separation can be measured by the stretching rate $\mu(t) \equiv(1 / t) \ln [R(t) / R(0)]$. It is assumed that the reduced dynamics (8) - (10) is ergodic. There is currently no rigorous proof of ergodicity. However, such an assumption relies on numerical evidence and on the following phenomenological argument. The deterministic loops described in Section [1] are randomly initiated by the near-origin behavior of the system, providing a mechanism of rapid memory loss that might ensure ergodicity. With this assumption, the time averages converge to ensemble averages, so that

$$
\mu(t)=\frac{1}{t} \int_{0}^{t} \sigma_{1}\left(t^{\prime}\right) \mathrm{d} t^{\prime} \rightarrow\left\langle\sigma_{1}\right\rangle \quad \text { as } t \rightarrow \infty .
$$

In other words, the distance between particles asymptotically behaves as $R(t)=R(0) \exp (t \lambda)$, where $\lambda=\left\langle\sigma_{1}\right\rangle$ is a non-random quantity referred to as the Lyapunov exponent. A positive Lyapunov exponent implies that the particle dynamics is chaotic [19].

Figure 5 shows numerical measurements of the Lyapunov exponent $\lambda$. The exponent remains positive for all values of the Stokes number. This means in particular that particles suspended in incompressible flow cannot experience strong clustering, which consists in the convergence of all trajectories together to form point clusters. This contrasts with the case of compressible flows where, for suitable values of St and of the compressibility, negative Lyapunov exponents are observed [24]. A first attempt to derive an analytic expression for $\lambda(\mathrm{St})$ was proposed by Piterbarg [14]. His approach is based on studying the Laplace transform $\varphi(p)$ of the distribution of the complex random variable $z=\sigma_{1}+i \sigma_{2}$, i.e. $\varphi(p, t)=\langle\exp (-p z(t))\rangle$ which satisfies

$$
\partial_{t} \varphi=-(p / \tau) \partial_{p} \varphi+p \partial_{p}^{2} \varphi-\left(2 D_{1} / \tau\right) p^{2} \varphi
$$

If $\varphi(p, t)$ reaches a steady state at large times, one can infer an analytic expression for the asymptotic solution $\varphi_{\infty}(p)$ by requiring that the right-hand side of (19) vanishes. It is then straightforward to deduce that the Lyapunov exponent satisfies $\lambda=-\lim _{p \rightarrow 0} \Re\left\{\partial_{p} \varphi_{\infty}\right\}$. This implies

$$
\lambda=-\frac{D_{1}}{2 \mathrm{St}} \Re\left\{1+\frac{\mathrm{Ai}^{\prime}(x)}{\sqrt{x} \operatorname{Ai}(x)}\right\}, x=(16 \mathrm{St})^{-2 / 3},
$$

where $\mathrm{Ai}$ and $\mathrm{Ai}^{\prime}$ designate the Airy function of the first kind and its derivative respectively. This prediction is compared to the numerical measurements in Fig. 5 . As stressed in 25], there is evidence that the moments $\varphi(p, t)$ do not converge to a steady state, but rather diverge at large times. This might explain the discrepancies observed in Fig. 5. However, the numerical precision is not high enough to test the presence of corrections to the analytic expression (20).

At large but finite time $t$, the distance between the two particles is measured by the stretching rate $\mu(t)=$ $(1 / t) \ln [R(t) / R(0)]$. This quantity becomes more and more sharply distributed around the Lyapunov exponent $\lambda$ as $t$ increases. More precisely, it obeys a large deviation principle and its pdf $p(\mu, t)$ takes the asymptotic form (see, e.g., [2])

$$
\frac{1}{t} \ln p(\mu, t) \sim-H(\mu),
$$

where $H$ is a positive convex function attaining its minimum in $\mu=\lambda$, in particular $H(\lambda)=0$. The rate function $H$ measures the large fluctuations of $\mu$, which are important to quantify particle clustering. Rate functions obtained from numerical experiments are represented in Fig. 5 for various values of the Stokes number. The function becomes less and less broad when St increases, a phenomenon that can be quantified in the limit St $\rightarrow \infty$ as discussed in Section VII Note that the same qualitative behavior is also observed for heavy particles suspended in homogeneous isotropic flow [27].

We now turn to the case of particles suspended in nondifferentiable flows $(h<1)$. As we dropped the boundary condition, the initial inter-particle distance $R(0)$ is the only relevant length scale. By using $R(0)$ instead of $L$ in the change of variables (5) - (7) the problem of relative dispersion is expressed solely in terms of the the Hölder exponent $h$ and of a time-dependent Stokes number which can be defined in terms of the local Stokes number as $\mathrm{St}_{t}=D_{1} \tau /[R(t)]^{2(1-h)}$. In particular, the evolution of $R(t)$ directly follows from the initial its value $\mathrm{St}_{0}$. From the evolution equation (10) for the reduced separation $\rho(t)=[R(t) / R(0)]^{1-h}$, we obtain

$$
\rho(t)=1+(1-h) \int_{0}^{t} \sigma_{1}\left(t^{\prime}\right) \mathrm{d} t^{\prime},
$$




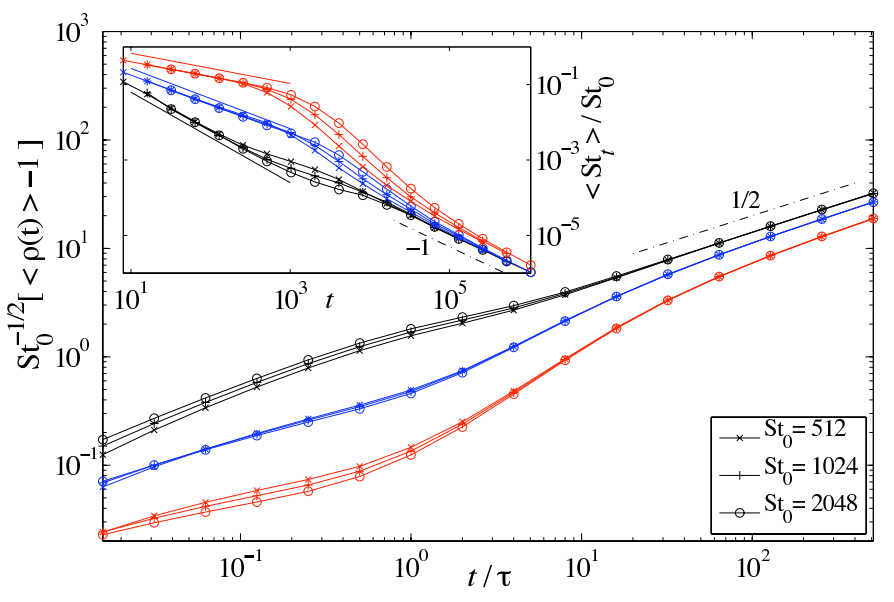

FIG. 6: Time evolution of the average rescaled separation $\langle(\rho(t)-\rho(0))\rangle$ for different initial Stokes numbers $\mathrm{St}_{0}$, and $h=0.4,0.6$, and 0.8 (from top to bottom). Inset: longtime behavior of the time-dependent Stokes number $\mathrm{St}_{t}=$ $D_{1} \tau / \rho^{2}(t)$ for different $\mathrm{St}_{0}$ and the same three values of $h$ (now from bottom to the top). The segments on the left indicate the slopes $-2 /(1-h)$ corresponding to the regime of ballistic separation.

where $\rho(\in[0, \infty))$ typically increases with time. The timedependent Stokes number $\mathrm{St}_{t}=D_{1} \tau / R^{2(1-h)}=\mathrm{St}_{0} / \rho^{2}$, which measures the effect of inertia when the particles are at a distance $R(t)$, decreases with time. Hence, conversely to the case of differentiable carrier flow, $\sigma_{1}$ is not a stationary process and the integral in (22) does not tend to $t\left\langle\sigma_{1}\right\rangle$.

Hereafter, we confine the discussion to the case $\mathrm{St}_{0} \gg 1$ because it contains a richer physics than smaller $\mathrm{St}_{0}$. As observed from Fig. 66, we can distinguish two regimes in the time behavior of $\rho(t)$. At first the particle separation evolves ballistically, i.e. $R(t) \propto t$, meaning that the timedependent Stokes number $\mathrm{St}_{t}$ decreases as $t^{-2 /(1-h)}$ (see inset of Fig. (6) and reaches order-unity values for $t \approx \tau$. During this phase, the time growth of $\rho$ is accelerated or slowed down and ultimately reaches a diffusive behavior $\propto t^{1 / 2}$. This corresponds to the limit of tracers, which is approached when $\mathrm{St}_{t} \ll 1$. At this stage, the inter-particle distance behaves as $R(t) \propto t^{1 / 2(1-h)}$ and, consequently, the $\mathrm{St}_{t}$ decreases as $1 / t$ (see Fig. 6).

The convergence to tracer diffusion in the limit of large distances $R$ gives an original way to interpret Richardson's law for delta-correlated velocity fields in terms of the asymptotic behavior of the reduced variables (5) - (7). When $\rho$ is large, the quadratic terms in the drift of equation (81) can be neglected and $\sigma_{1}$ behaves as an OrnsteinUhlenbeck process with response time $\tau$. However, when $\sigma_{1}$ becomes of the order of $\rho /(h \tau)$, the quadratic terms cease to be negligible and they push the trajectory back to $\sigma_{1}>0$. This process happens on time scales that are of the order of unity and thus much smaller than the time scales relevant for large-scale dispersion. Hence the dynamics of $\sigma_{1}(t)$ can be approximated as an Ornstein-

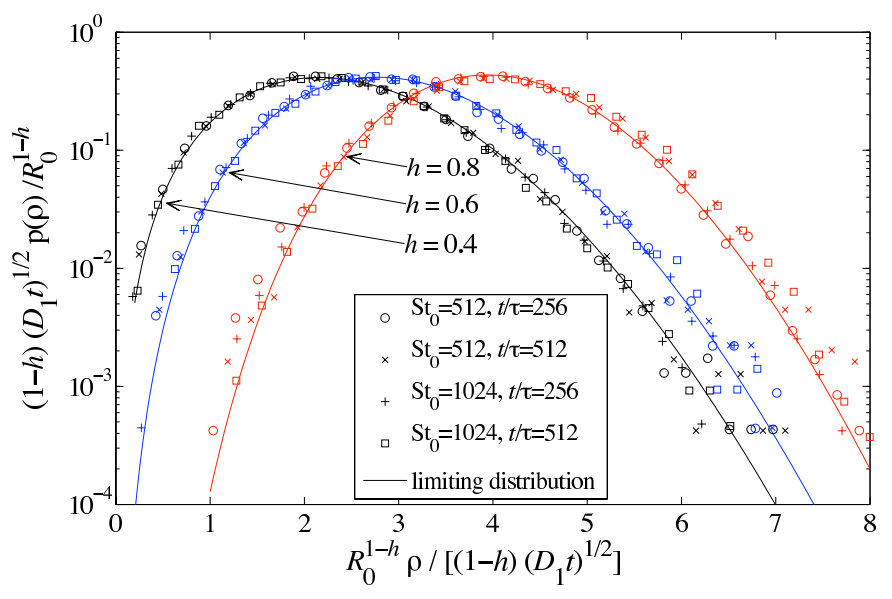

FIG. 7: Pdf of the rescaled separation $\rho(t)$ for various combinations of $\mathrm{St}_{0}$ and large times $t$. The solid lines represent the limiting distribution given by (23) with $A=1 / 4$.

Uhlenbeck process with reflective boundary condition on $\sigma_{1}=\rho /(h \tau)$. This implies that $\rho$ has a diffusive behavior. More specifically, numerical simulations (see Fig. (7) show that the pdf of $\rho$ behaves as

$$
p(\rho, t) \propto \rho^{\nu} t^{-(\nu+1) / 2} \exp \left[-A \rho^{2} / t\right],
$$

where $\nu=(1+h) /(1-h)$ and $A$ is a positive constant. At large times and consequently large distances $\mathrm{St}_{t} \rightarrow 0$, the tracer limit is fully recovered as confirmed by expressing the above relation in terms of the physical distance $R=$ $\rho^{1 /(1-h)}$. Indeed it becomes identical to the law that governs the separation of tracers in a Kraichnan flow [28]. However, a direct derivation of (23) in terms of the $\rho$ and $\boldsymbol{\sigma}$ dynamics is still lacking.

\section{EXACT RESULTS IN ONE DIMENSION}

A number of analytical results were derived for onedimensional flows [29, 30, 31]. Although such flows are always compressible, their study helps improving the intuition for the dynamics of inertial particles in higherdimensional random flows. In particular, several results on caustic formation hold also in two-dimensional (incompressible) flows because the typical velocity fluctuations, which lead to caustic formation, are effectively one-dimensional.

Here, we focus on one-dimensional smooth flows, for which the equations analogous to (8) - (10) reduce to

$$
\begin{aligned}
\dot{\sigma} & =-\sigma / \tau-\sigma^{2}+\sqrt{C} \eta(t), \\
\dot{R} & =\sigma R,
\end{aligned}
$$

where $\sigma=V / R$ and, as in (8)-(10), $C=2 D_{1} / \tau^{2}$. The quadratic term in (24) implies that $\sigma$ can escape to $-\infty$ with a finite probability. These events are the onedimensional counterpart of the loops described in Sec- 
tion $\amalg$ and correspond to the formation of caustics: particle trajectories intersect with a finite relative velocity. Note that the equation for $\sigma$ decouples from the equation for $R$, so that it can be studied separately. Stationary statistics of $\sigma$ can be described by the pdf $P(\sigma)$ which obeys the one-dimensional Fokker-Planck equation

$$
\left[\partial_{\sigma}\left(\sigma / \tau+\sigma^{2}\right)+(C / 2) \partial_{\sigma}^{2}\right] P(\sigma)=0 .
$$

This equation can be rewritten as $\partial_{\sigma} J(\sigma)=0$, where $J(\sigma)=\left(\sigma / \tau+\sigma^{2}\right) P(\sigma)+C P^{\prime}(\sigma) / 2$ is a probability flux in the $\sigma$-space. Equation (26) is supplied by the boundary conditions $J(+\infty)=J(-\infty)$, which are required to resolve escapes to infinity and thus caustic formations. Indeed such events correspond to particle crossings during which $R \rightarrow 0$ and $V$ remains finite, so that $\sigma=V / R$ changes sign. Hence, all particles escaping to $\sigma=+\infty$ reappear at $\sigma=-\infty$. The stationary solutions of Eq. (26) satisfying such a boundary condition corresponds to a constant flux $J$ and can be written as

$$
P(\sigma)=\frac{2|J|}{C} \mathrm{e}^{-2 U(\sigma) / C} \int_{-\infty}^{\sigma} \mathrm{d} \sigma^{\prime} \mathrm{e}^{2 U\left(\sigma^{\prime}\right) / C},
$$

where $U(\sigma)=\sigma^{3} / 3+\sigma^{2} / 2 \tau$. Note that as in two dimensions, $P(\sigma)$ has power-law tails. The argument presented in Section II can actually be straightforwardly applied with the difference that there is no loop anymore but just escapes to infinity occurring with a probability that is independent of $\sigma$. This leads to $P(\sigma) \propto|\sigma|^{-2}$ for $|\sigma| \rightarrow \infty$ (the exponent is actually $-(1+1 / h)$ in the general case of Hölder-continuous carrier flows).

Using the constant-flux solution (27), one can derive the Lyapunov exponent $\lambda=\langle\sigma\rangle$. As shown in [30], its value non-trivially depends on the Stokes number. For $\mathrm{St}=D_{1} \tau \ll 1$, it is negative and behaves like $\lambda \simeq-D_{1}$ while for St $\gg 1$ it becomes positive and its value is given by the asymptotic expression $\lambda \simeq D_{1} \mathrm{St}^{-2 / 3} \sqrt{3} 12^{5 / 6} \Gamma(5 / 6) /(24 \sqrt{\pi})>0$. There exists a critical value of the Stokes number $(\approx 0.827)$ for which the Lyapunov exponent changes its sign. This phenomenon of sign-changing has been dubbed path coalescence transition by Wilkinson and Mehlig in [30]. It is closely related to the aggregation-disorder transition discussed in [29]. The sign of the Lyapunov exponent determines how the distance between two initially close particles evolves with time. It turns out that the answer depends on the particle size: small particles (with smallenough Stokes numbers) tend to approach each other, while large particles (with large Stokes numbers) get separated by the flow.

Another important phenomenon which was extensively studied within the one-dimensional model is the formation of caustics. The average rate of caustics formation is given by the absolute value of the probability flux $J$. For large values of the Stokes number it can be written as $|J| \simeq D_{1} \mathrm{St}^{-2 / 3} \Gamma(5 / 6) 12^{5 / 6} /\left(8 \pi^{3 / 2}\right)$, while for small Stokes it becomes exponentially small
$|J| \sim D_{1}(2 \pi \mathrm{St})^{-1} \exp [-1 /(6 \mathrm{St})]$. The formation of caustics is a stochastic process, whose properties can be described by the pdf of the caustic formation time $T$. In 31] it is shown that for St $\ll 1$ this pdf can be estimated as $P(T) \propto \exp [-1 /(6 \mathrm{St})]$ for $\tau \ll T \ll \tau \exp [1 /(6 \mathrm{St})]$ and $P(T) \propto \exp \left[-w /\left(3 C T^{3}\right)\right]$, with $w=\Gamma(1 / 4)^{8} / 96 \pi^{2}$ ( $\Gamma$ denoting the Gamma-function here), for $T \ll \tau$. The exponential factor $\exp [-1 /(6 \mathrm{St})]$ which characterizes the small rate of caustic formations for St $\ll 1$ can be easily explained if one formally considers Eq. (24) as a Langevin equation for a particle which is driven by the thermal noise $\eta(t)$ and evolves in the potential $U(\sigma)$. In this case, the rate of caustic formation is given by the probability for the particle to tunnel through the potential barrier in $U(\sigma)$. Such probability can be estimated as $\exp [-1 /(6 \mathrm{St})]$. For large Stokes numbers, the barrier disappears and the rate of caustic formation is not exponentially damped anymore.

\section{SMALL STOKES NUMBER ASYMPTOTICS}

This Section reports some asymptotic results related to the limit of small particle inertia. The first part summarizes the approach developed by Mehlig, Wilkinson, and collaborators for differentiable flows $(h=1)$. In analogy to the WKB approximation in quantum mechanics (see, e.g. [32]), the authors construct perturbatively the steady solution to the Fokker-Planck equation associated to the reduced system (8) - (9). In the second part of this Section original results are reported where the particle dynamics is approximated as the advection by a synthetic flow comprising an effective compressible drift which accounts for leading-order corrections due to particle inertia.

Mehlig and Wilkinson proposed in 24] (see also 33]) to approach the limit of small Stokes numbers in terms of the variables $x_{1}=\left(\tau / D_{1}\right)^{1 / 2} \sigma_{1}$ and $x_{2}=\left(\tau / 3 D_{1}\right)^{1 / 2} \sigma_{2}$. From equations (8) (9), their time evolution follows to satisfy

$$
\begin{aligned}
& \dot{x}_{1}=-x_{1}-\varepsilon\left[x_{1}^{2}-3 x_{2}^{2}\right]+\sqrt{2} \eta_{1}(s), \\
& \dot{x}_{2}=-x_{2}-2 \varepsilon x_{1} x_{2}+\sqrt{2} \eta_{2}(s),
\end{aligned}
$$

where $\varepsilon=\sqrt{\mathrm{St}}$, dots denote derivatives with respect to the rescaled time $s=t / \tau$ and $\eta_{1}$ and $\eta_{2}$ are independent white noises. The evolution equations (28) $-(29)$ can be written in vectorial form, namely $\dot{\boldsymbol{x}}=-\boldsymbol{x}+\varepsilon \mathbf{V}(\boldsymbol{x})+\sqrt{2} \boldsymbol{\eta}$, where $\boldsymbol{x}=\left(x_{1}, x_{2}\right), \boldsymbol{\eta}=\left(\eta_{1}, \eta_{2}\right)$ and $\mathbf{V}$ denotes the quadratic drift. The steady-state probability density $p(\boldsymbol{x})$ is a solution to the stationary Fokker-Planck equation

$$
\nabla_{\boldsymbol{x}}^{2} p+\nabla_{\boldsymbol{x}} \cdot(\boldsymbol{x} p)=\varepsilon \nabla_{\boldsymbol{x}} \cdot[\mathbf{V}(\boldsymbol{x}) p] .
$$

Next step consists in writing perturbatively the probability density of $\boldsymbol{x}$ as $p(\boldsymbol{x})=\exp \left(-|\boldsymbol{x}|^{2} / 4\right)\left(Q_{0}+\varepsilon Q_{1}+\right.$ 
$\left.\varepsilon^{2} Q_{2}+\cdots\right)$. The functions $Q_{k}$ satisfy the recursion relation $\mathcal{H}_{0} Q_{k+1}=\mathcal{H}_{1} Q_{k}$, where

$$
\begin{aligned}
& \mathcal{H}_{0}=1+\nabla_{\boldsymbol{x}}^{2}-|\boldsymbol{x}|^{2} / 4, \\
& \mathcal{H}_{1}=\nabla_{\boldsymbol{x}} \cdot \mathbf{V}(\boldsymbol{x})+\boldsymbol{x} \cdot \mathbf{V}(\boldsymbol{x}) / 2 .
\end{aligned}
$$

The operator $\mathcal{H}_{0}$ is the Hamiltonian of an isotropic twodimensional quantum harmonic oscillator. This suggests introducing creation and annihilation operators and to expand the functions $Q_{k}$ in terms of the eigenstates of the harmonic oscillator (see [24, 33] for details).

This approach yields a perturbative expansion of the Lyapunov exponent 24]

$$
\lambda=D_{1}\left\langle x_{1}\right\rangle / \varepsilon=2 D_{1} \sum_{k \geq 0} a_{k} \varepsilon^{2 k}=2 D_{1} \sum_{k \geq 0} a_{k} \mathrm{St}^{k},
$$

where the coefficients $a_{k}$ satisfy the recurrence relation

$$
a_{k+1}=4(4-3 k) a_{k}-2 \sum_{\ell=0}^{k} a_{\ell} a_{k-\ell},
$$

with $a_{0}=1$. For large $k$, these coefficients behave as $a_{k} \sim(-12)^{k} k$ !, so that the series (33) diverges no matter how small the value of $\varepsilon$ (and thus of St). Hence the sum representation of $\lambda$ makes sense as an approximation only if truncated at an index $k_{\star}$ for which $\left|a_{k} \mathrm{St}^{k}\right|$ attains its minimum. For small values of St, $k_{\star} \sim 1 /(12 \mathrm{St})$ and the error of the asymptotic approximation is of the order of the smallest term, namely $\sim\left|a_{k_{\star}} \mathrm{St}^{k_{\star}}\right| \sim \exp [-1 /(12 \mathrm{St})]$. This approach was refined by Wilkinson et al. [33] adopting an approach based on Padé-Borel summation, which was found to yield satisfactory results.

The non-analyticity of $\lambda(\mathrm{St})$ at $\mathrm{St}=0$ is interpreted in [24] as a drawback of the perturbative approach. Indeed the quadratic terms in (28) - (29) are not negligible for all values of $x_{1}$ and $x_{2}$ : When $|\boldsymbol{x}|$ becomes larger than $\varepsilon^{-1}$ they are actually dominant and the trajectory performs a loop in the $\boldsymbol{x}$ (or $\boldsymbol{\sigma}$ ) plane (see Section $\amalg$ ). When $\mathrm{St}=\varepsilon^{2}$ is small, the probability to initiate such a loop is given by the tail of the distribution governing scales $|\boldsymbol{x}| \ll \varepsilon^{-1}$, and is hence $\propto \exp \left[-1 /\left(6 \varepsilon^{2}\right)\right]$, which coincides with the one-dimensional result discussed in previous Section, confirming the relevance of $d=1$ physics to the formation of caustics in higher dimension. Taking into account this correction due to caustics, i.e. the contribution of events when the particles approach very close to each other keeping a finite velocity difference, Mehlig and Wilkinson proposed to write the Lyapunov exponent as

$$
\lambda / D_{1} \sim B \mathrm{St}^{-1} \mathrm{e}^{-1 /(6 \mathrm{St})}+2 \sum_{k=0}^{k_{\star}} a_{k} \mathrm{St}^{k},
$$

where $B$ is a positive constant. We finish this summary by stressing that this approach equally applies to the case of compressible carrier flows [24], and was extended to three dimensions where it yields a prediction on the Stdependence of the three largest Lyapunov exponents [33].
The above perturbative approach can be generalized to small particles evolving in rough flows. For small (local) Stokes numbers, the characteristic time scales of velocity evolution are much smaller compared to the temporal scales associated to the dynamics of the particle separation. Therefore, one can obtain the effective equation for the evolution of particle separation by averaging over the fast velocity difference variables. The systematic mathematical strategy of such an averaging was proposed in 34 in the context of stochastic climate models. This strategy is closely related to the Nakajima-Zwanzig technique which was developed to study similar problems arising in damping theory [35, 36]. Applications of this technique to the elimination of fast variables in Fokker-Planck equations are discussed in [37, 38]. In this framework one can derive an expansion for the Fokker-Planck type operator entering into the equation for the slow-variable probability distribution function. In our case, this leads to a closed equation for the pdf of the particle separation $R$. This equation can be used to determine the local correlation dimension $d_{2}(r)$ for $\operatorname{St}(r) \ll 1$. We present here only the general idea and the main results; details of the calculations will be reported elsewhere.

To carry out the above-mentioned procedure the joint position-velocity pdf $p(\boldsymbol{r}, \boldsymbol{v})$ is approximated by

$$
p(\boldsymbol{r}, \boldsymbol{v}) \simeq p(\boldsymbol{r}) P_{\boldsymbol{r}}(\boldsymbol{v})+\tilde{p}(\boldsymbol{r}, \boldsymbol{v}),
$$

where $\tilde{p}(\boldsymbol{r}, \boldsymbol{v})$ denotes subleading terms which are $\mathrm{O}(\mathrm{St})$; $P_{\boldsymbol{r}}(\boldsymbol{v})$ is the stationary distribution associated to the fast velocity variables and satisfies the Fokker-Planck equation

$$
\hat{L}_{0} P_{\boldsymbol{r}}(\boldsymbol{v}) \equiv-\left[\frac{1}{\tau} \partial_{v}^{i} v^{i}+\frac{b^{i j}(\boldsymbol{r})}{\tau^{2}} \partial_{v}^{i} \partial_{v}^{j}\right] P_{\boldsymbol{r}}(\boldsymbol{v})=0,
$$

with the normalization condition $\int \mathrm{d} \boldsymbol{v} P_{\boldsymbol{r}}(\boldsymbol{v})=1$. Without loss of generality, it is assumed that the subleading terms $\tilde{p}(\boldsymbol{r}, \boldsymbol{v})$ in the approximation (36) do not contribute to the normalization condition, so that $\int \mathrm{d} \boldsymbol{v} p(\boldsymbol{r}, \boldsymbol{v})=$ $p(\boldsymbol{r})$. The effective equation for $p(\boldsymbol{r})$ can be derived by introducing the expansion $p(\boldsymbol{r})=\sum_{k=0}^{\infty} \mathrm{St}^{k / 2} p_{k}(\boldsymbol{r})$. This expansion, which enters the definition (36), is then substituted into (4) and all terms of the same order in St are collected. Note, that the operator $\hat{L}_{1}=\partial_{r}^{i} v^{i}$ entering Eq. (4) is smaller than the other operators by a factor $\mathrm{St}^{1 / 2}$. The chain of equations for $p_{k}(\mathbf{r})$ has a solvability condition that results in the following effective equation for $p(\boldsymbol{r})$ :

$$
\left(\hat{M}_{1}+\hat{M}_{2}+\cdots\right) p(\boldsymbol{r})=0,
$$

where the operators $\hat{M}_{k}$ can be written as

$$
\hat{M}_{k} p(\boldsymbol{r})=\int \mathrm{d} \boldsymbol{v}\left(\hat{L}_{1} \hat{L}_{0}^{-1}\right)^{k} \hat{L}_{1} p(\boldsymbol{r}) P_{\boldsymbol{r}}(\boldsymbol{v}) .
$$

$\hat{L}_{0}^{-1}$ denotes here the inverse of $\hat{L}_{0}$, i.e. the Green function obtained from (37) with the right-hand side replaced 
by a $\delta$ function. This operator is defined in such a way that $\int \mathrm{d} \boldsymbol{v} \hat{L}_{0}^{-1} f(\boldsymbol{v})=0$ for any function $f(\boldsymbol{v})$ satisfying $\int \mathrm{d} \boldsymbol{v} f(\boldsymbol{v})=0$. One can check that the leading-order operator is $\hat{M}_{1}=\partial_{r}^{i} b^{i j}(\boldsymbol{r}) \partial_{r}^{j}$ which, as expected, corresponds to turbulent diffusion. Indeed the dynamics of tracers is recovered when $\mathrm{St} \rightarrow 0$. The pdf $p(\boldsymbol{r})$ which solves the equation $\hat{M}_{1} p(\boldsymbol{r})=0$ is simply the uniform distribution. To measure particle clustering, which can be estimated for instance by the local correlation dimension $d_{2}(r)$ (see Section III), one has to calculate the next order operators. It can be easily checked that all operators $\hat{M}_{k}$ of even order $k$ are zero. The first non-vanishing correction to $\hat{M}_{1}$ is thus given by the third order operator $\hat{M}_{3}$. When interested in the stationary distribution only, the terms which enter this operator and which are associated to transients can be disregarded and one can write:

$$
\hat{M}_{3} \cdot=\partial_{r}^{i}\left[V^{i} \cdot\right], \text { with } V^{i}=-\frac{1}{2}\left(\partial_{r}^{k} \partial_{r}^{l} b^{i j}\right)\left(\partial_{r}^{j} b^{k l}\right) \text {. }
$$

The operator $\hat{M}_{3}$ can be interpreted as an effective drift in $\boldsymbol{r}$-space and, for the Kraichnan model, represented as $V^{i}=-2\left(d^{2}-1\right)(d-2+4 h) h^{2} \mathrm{St}^{2}(r) r^{i}$. The functional form of this drift implies that the first non-vanishing corrections to the uniform distribution are proportional to $\mathrm{St}(r)$. Indeed, for isotropic flows one can look for a solution $p(\boldsymbol{r})$, which depends only on the modulus $r$ of its argument. In this case Eq. (38) becomes an ordinary differential equation of Fokker-Planck type. Looking for a non-flux solution one readily obtains the desired $p(r)$. In rough flows $(h<1)$, one has $\ln p(\boldsymbol{r}) \sim$ $\left[(d+1)(d-2+2 h) h^{2} /(1-h)\right] \mathrm{St}(r)$ and the local correlation dimension behaves as

$$
d_{2}(r) \simeq d-\frac{2 d(d+1)(d-2+4 h) h^{2}}{d-2+2 h} \operatorname{St}(r) .
$$

Note that the second term on the right-hand side of the above expression disappears for $h \rightarrow 0$, confirming once again the finding of the previous Sections about the decrease of clustering going from smooth to rough flows. For differentiable carrier flows $(h=1)$, the distribution has algebraic tails: $\ln p(\boldsymbol{r}) \sim-2(d+1)(d+2) \operatorname{St} \ln r$, and hence the correlation dimension behaves as

$$
\mathcal{D}_{2}=d-2(d+1)(d+2) \mathrm{St}+\mathrm{O}\left(\mathrm{St}^{2}\right) .
$$

The dimension deficit $d-\mathcal{D}_{2}$ is equal to $24 \mathrm{St}$ for twodimensional flows and to $d-\mathcal{D}_{2}=40$ St for threedimensional ones. The latter result is in agreement with the dimension deficit of the Lyapunov dimension reported by Wilkinson et al. in 33]. The above predictions on the dimension deficit, for smooth flows, are in very good agreement with numerical simulations in two and three dimensions, see Fig. 8. We conclude this Section by noticing that in time-correlated random smooth flows, as well as in developed turbulence, the dimension deficit has been shown to be $\propto \mathrm{St}^{2}[5,11,39,40]$. Therefore, including temporal correlations seems to be crucial to reproduce the details of the small-Stokes statistics of turbulent suspensions.

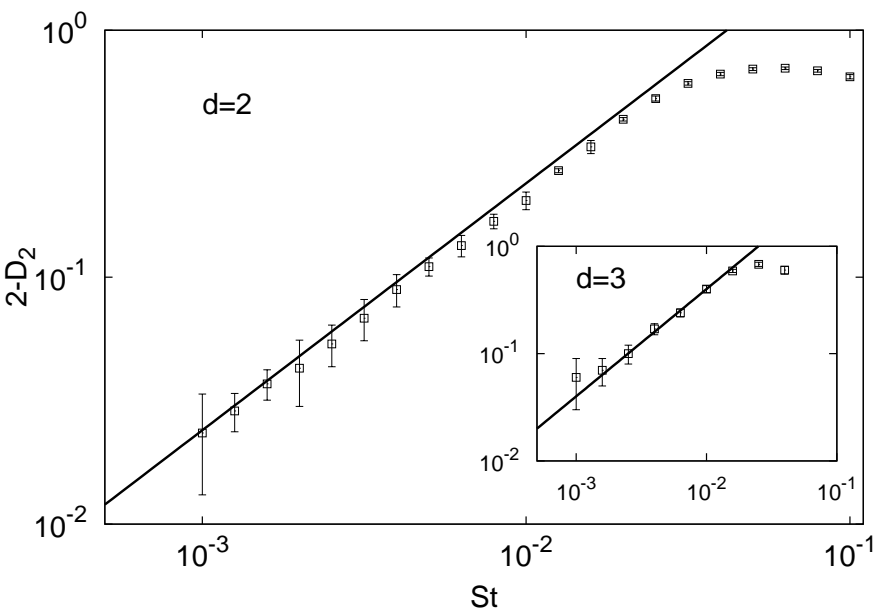

FIG. 8: Dimensional deficit $2-\mathcal{D}_{2}$ versus St in $d=2$ for smooth flows $(h=1)$. Inset: same for $d=3$. Points represent numerical results and the straight line corresponds to the perturbative predictions given by (42) for $d=2$ and 3 respectively.

\section{LARGE STOKES NUMBER ASYMPTOTICS}

Particles with huge inertia (St $\gg 1$ ) take an infinite time to relax to the velocity of the carrier fluid. They become therefore uncorrelated with the underlying flow and evolve with ballistic dynamics, moving freely and maintaining, almost unchanged, their initial velocities. This limit is particularly appealing for deriving asymptotic theories [16]. In this Section, we focus on two aspects, namely the problem of the recovery of homogeneous/uniform distribution for St $\gg 1$ and the problem of the asymptotic scaling for the statistics of the particle separation and of the velocity differences.

\section{A. Saturation of the correlation dimension}

Ballistic particles injected homogeneously and uniformly remain so [41]. Hence for the correlation dimension associated with their distribution (13) one has $\mathcal{D}_{2}=d$. This result follows directly from the FokkerPlanck equation (4), which can be seen as an advectiondiffusion equation in phase space. The effective flow is compressible because of the term $-\partial_{v} v / \tau$ but, in the limit $S t \rightarrow \infty$, it becomes negligible and the equation reduces to diffusion plus advection by an incompressible flow. The resulting stationary pdf is thus uniform in phase space and hence in its projection in position space. Moreover, as particle velocities and fluid flow are uncorrelated and consequently the particles are not correlated with each other, the exponent $\Gamma$ which characterizes the small-scale behavior of the approaching rate (see Section III) vanishes. Thus $\mathcal{D}_{2} \rightarrow d$ and $\Gamma \rightarrow 0$ for $\mathrm{St} \rightarrow \infty$.

This asymptotic regime can be achieved via two possible scenarios: (a) asymptotic convergence of $\mathcal{D}_{2}$ to $d$, and 
(b) saturation of $\mathcal{D}_{2}$ to $d$ for Stokes numbers above a critical value $\mathrm{St}^{\dagger}$. In what follows, we provide evidence for (b), limiting the discussion to two-dimensional smooth flows.

Let us first discuss a phenomenological argument in favor of saturation. As already noted in Section III their dissipative dynamics yields the phase-space trajectories of the particles to converge onto a random, dynamically evolving attractor, which is typically characterized by a multifractal measure [19, 20]. In our setting, this measure is the phase-space correlation dimension defined in equation (14). Ballistic motion for $\mathrm{St} \gg 1$ corresponds to $\overline{\mathcal{D}}_{2} \rightarrow 2 d$, therefore a critical Stokes number $\mathrm{St}^{\dagger}$ exists such that $\overline{\mathcal{D}}_{2}\left(\mathrm{St}^{\dagger}\right)=d$. The particles' spatial distribution is obtained by projecting the $(2 \times d)$-dimensional phase space onto the $d$-dimensional physical space. It is tempting to apply a rigorous result on the projection of random fractal sets [22, 42] stating that for almost all projections, the correlation dimension of the projected set is related to that of the unprojected one via the relation

$$
\mathcal{D}_{2}=\min \left\{d, \overline{\mathcal{D}}_{2}\right\}
$$

Having $\overline{\mathcal{D}}_{2}\left(\mathrm{St}^{\dagger}\right)=d$ with the above expression implies that $\mathcal{D}_{2}(\mathrm{St})=d$ for all $\mathrm{St} \geq \mathrm{St}^{\dagger}$. Unfortunately, there is a priori no reason for assuming some kind of isotropy in phase space which justifies the validity of (43). We thus proceed numerically.

As Eq. (43) requires the isotropy of the set, we have tested whether this applies to our case. The correlation dimension of different two-dimensional projections was evaluated through the computation of the probabilities $P_{2}^{\alpha, \beta}(r)$ of having two particles at a distance less than $r$ using the norm $\Delta_{\alpha, \beta}^{2}=\delta_{\alpha}^{2}+\delta_{\beta}^{2}$, with $\alpha, \beta=$ $X, Y, V_{X} / D_{1}, V_{Y} / D_{1}$, and $\delta_{\alpha}$ denoting the coordinate- $\alpha$ separation between the two particles. Note that $\alpha=X$ and $\beta=Y$ corresponds to the spatial correlation dimension discussed so far. Figure 9 shows the logarithmic derivatives $\left(\mathrm{d} \ln P_{2}^{\alpha, \beta}(r)\right) /(\mathrm{d} \ln r)$ for various $\alpha, \beta$ and three different values St. All curves collapse within errorbars, confirming that the projection is rather typical and thus strengthening the argument in favor of saturation. However, as can be seen in Fig. 9, the logarithmic derivatives on the different projections are curved, indicating behaviors different from the expected power law. It is therefore difficult to decide whether or not the saturation occurs. As discussed in [44], one can understand the curvature of the local slopes with the presence of sub-dominant terms, e.g., with the superposition of two power laws $P_{2}(r) \simeq A r^{a}+B r^{b}$. In our case, one can expect that

$$
P_{2}(r)=A r^{\overline{\mathcal{D}}_{2}}+B r^{d}
$$

where $d$ and $\overline{\mathcal{D}}_{2}$ are the only dimensions entering the problem [16]. For $\overline{\mathcal{D}}_{2}<d$, the second power law can be interpret also as the contribution of caustics [18, 26]: With non-zero probability, particles may be very close to

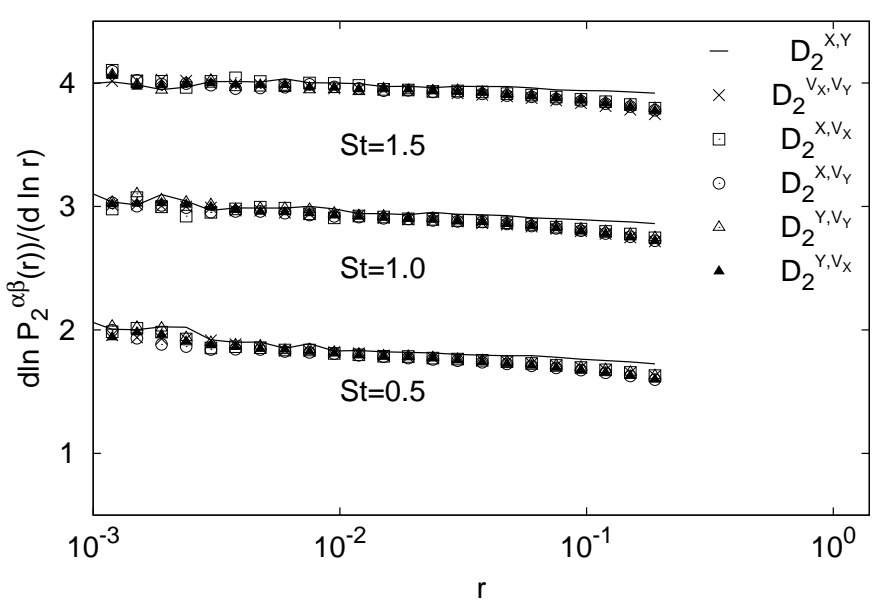

FIG. 9: Logarithmic derivative $\left(\mathrm{d} \ln P_{2}^{\alpha, \beta}(r)\right) /(\mathrm{d} \ln r)$ for different projections $\alpha, \beta$ for $\mathrm{St}=0.5$, St $=1$ (shifted up by a factor 1 ), and $\mathrm{St}=1.5$ (shifted up by a factor 2 ). A small mismatch in the scaling range can observed for large $r$ (this is unavoidable as positions and velocities involve different scales).

each other with quite different velocities, see Section V. Once projected onto physical space, caustics appear as spots of uncorrelated particles, and hence, the correlation dimension is locally $\mathcal{D}_{2}=d$. The validity of (44) as well as of the projection formula (43) was confirmed in Ref. [16], .

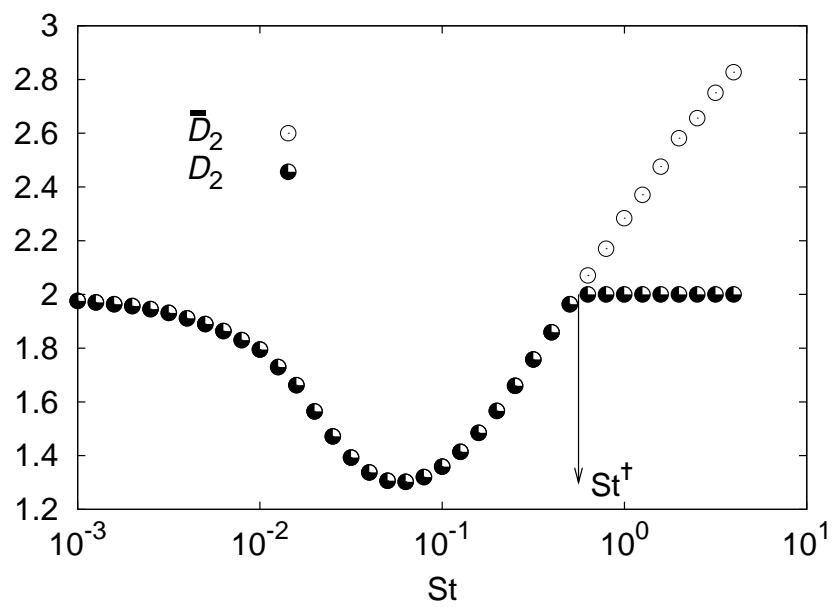

FIG. 10: Physical space $\mathcal{D}_{2}$, and phase-space $\overline{\mathcal{D}}_{2}$ correlation dimensions versus St as obtained by using (44) for fitting the exponents. Errors are of the order of the size of the symbol. The arrow indicates the estimated location of $S t^{\dagger}$.

Figure 10] summarizes the results depicted above. In particular, $\overline{\mathcal{D}}_{2}$ clearly displays a crossover to values larger than $d$ for $S t>\mathrm{St}^{\dagger} \approx 0.6$. $\mathcal{D}_{2}$, once properly estimated by using (43), displays the saturation to $d=2$ above $\mathrm{St}^{\dagger}$, at which the large Stokes asymptotics starts, at least for the particle distribution.

Let us comment briefly on the implication of saturation on the behavior of the approaching rate which, in the limit St $\rightarrow \infty$, is characterized by the exponent $\Gamma \rightarrow 0$. 
Similarly to $\mathcal{D}_{2}$, deviations of $\Gamma$ from its limiting value cannot be determined by scaling arguments. Saturation of $\mathcal{D}_{2}$ would however affect $\Gamma$. This is related to the dominant contribution of caustics which might imply also the saturation of $\Gamma$ to 0 for sufficiently large Stokes numbers. Though numerical experiments confirm this scenario [16], saturation cannot be studied with as much detail as for $\mathcal{D}_{2}$. At present, there is no simple phenomenological argument for the subleading terms as for $\mathcal{D}_{2}$.

\section{B. Scaling arguments}

The limit of large values of the Stokes number can be approached by assuming $\tau \rightarrow \infty$ and keeping $C=$ $2 D_{1} /\left(\tau L^{1-h}\right)^{2}$ constant. The dynamics (8) - (9) for the relative velocity differences can then be approximated by

$$
\begin{aligned}
& \dot{\sigma}_{1} \simeq-\left(h \sigma_{1}^{2}-\sigma_{2}^{2}\right) / \rho+\sqrt{C} \eta_{1} \\
& \dot{\sigma}_{2} \simeq-(h+1) \sigma_{1} \sigma_{2} / \rho+\sqrt{(1+2 h) C} \eta_{2} .
\end{aligned}
$$

For a given exponent $h$, the limiting dynamics depends solely on $C$ while - after non-dimensionalizing time and relative velocities by $\tau$ - the general dynamics depends on $\operatorname{St}(L)$ only (see the Introduction). This congruence, which was first used in 45] for determining the large-St behavior of the Lyapunov exponent, allows to derive scaling arguments of various other quantities characterizing two-particle dynamics.

Let us detail this for the distribution of the longitudinal velocity difference $\sigma_{1}$. It is clear from the above considerations that for fixed $h$ and $\sigma_{1} \gg(1 / \tau)$ the following relation holds

$$
\tau \tilde{p}\left(\tau \sigma_{1} ; \mathrm{St}\right) \simeq p\left(\sigma_{1} ; C\right) .
$$

Differentiating with respect to $D_{1}$ and $\tau$ gives a necessary condition for such a behavior: $p$ must satisfy

$$
p+\sigma_{1} \partial_{\sigma_{1}} p+3 C \partial_{C} p=0,
$$

which itself implies $p\left(\sigma_{1} ; C\right)=C^{-1 / 3} f\left(C^{-1 / 3} \sigma_{1}\right)$, so that

$$
p\left(\sigma_{1}\right) \simeq \mathrm{St}^{-1 / 3} \tau f\left(\mathrm{St}^{-1 / 3} \tau \sigma_{1}\right) \quad \text { for } \mathrm{St} \gg 1 .
$$

As shown in Fig. 11] this asymptotic scaling behavior can be observed numerically. As a consequence of (49), for differentiable carrier flows $(h=1)$ the Lyapunov exponent $\lambda=\left\langle\sigma_{1}\right\rangle$, which measures the asymptotic growth rate of the inter-particle distance (see Section IV]), behaves as

$$
\lambda \simeq c D_{1} \mathrm{St}^{-2 / 3} \quad \text { for } \mathrm{St} \gg 1,
$$

where $c$ is a parameter-independent positive constant. Note that the original derivation [45] of this law applies also to compressible carrier flows, so the constant $c$ depends on the compressibility of the fluid velocity field. It

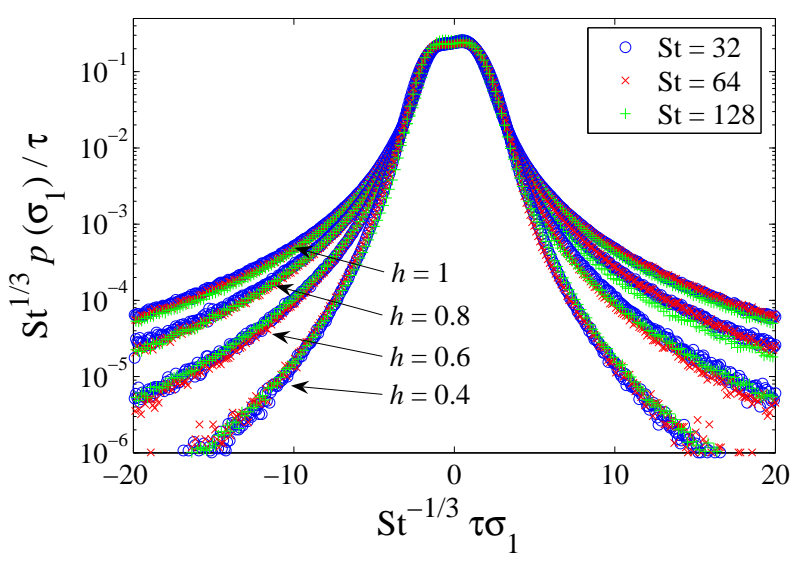

FIG. 11: Pdf of the non-dimensional longitudinal velocity difference $\sigma_{1}$ at large values St (symbols are for different values) for various values of $h$.

is shown in [16] that this result also holds in three dimensions. Its confirmation by numerical simulations is illustrated in Fig. 12,

The scaling argument described above can be carried forward to the fluctuations of the stretching rate $\mu(t)=(1 / t) \ln [R(t) / R(0)]$. As we have seen in Section [IV] for large times the distribution of $\mu$ obeys the large deviation principle (21). It can be shown (see [16] for details) that the associated rate function $H(\mu)=$ $\lim _{t \rightarrow \infty}(1 / t) \ln p(\mu, t)$ satisfies

$$
H(\mu) \simeq D_{1} \mathrm{St}^{-2 / 3} h\left(\mathrm{St}^{2 / 3} \mu / D_{1}\right) \quad \text { for } \mathrm{St} \gg 1 .
$$

This scaling is confirmed numerically (inset of Fig. 12).

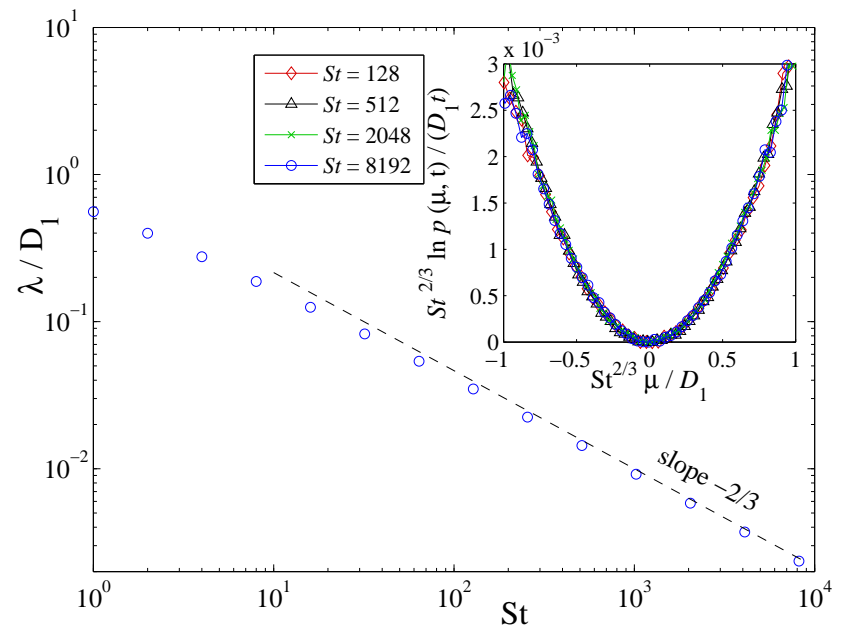

FIG. 12: Lyapunov exponent $\lambda$ versus St. The dashed line is the asymptotic prediction (50). Inset: rate function $H(\mu)$ for various large values of St.

We finally comment on how the stretching rate fluctuations change with St. Taylor expansion of $H$ around its 
minimum together with the scaling behavior (51) shows that the standard deviation of the stretching rate is of the order of $\mathrm{St}^{-1 / 3} / \sqrt{t}$. For a given time $t$, the stretching rate $\mu$ distributes more and more sharply around $\lambda$ when St increases. This behavior was anticipated by the numerical measurements reported in Section [V] and is observed in direct numerical simulations of heavy particles in homogeneous isotropic flows [27].

\section{REMARKS AND CONCLUSIONS}

Before concluding this paper the results discussed so far are commented in the light of what is known about real turbulent suspensions, which are relevant to most applications. Let us start by recalling the main features of turbulent flows. Turbulence is a multi-scale phenomenon [46] which spans length scales ranging from a large (energy injection) scale $L$ to the very small (dissipative) scale $\eta$, often called the Kolmogorov scale. This hierarchy of length scales is associated with a hierarchy of time scales: from the large-scale eddy turnover time $\tau_{L}$ to the Kolmogorov time $\tau_{\eta}$. Both ratios $L / \eta$ and $\tau_{L} / \tau_{\eta}$ increase with the Reynolds number Re of the turbulent flow. Therefore, in general settings, no separation of time scales can be invoked to simplify the motion of suspended particles. However, in two circumstances simplifications are possible, namely:

(i) For particles with a response time $\tau$ much greater than $\tau_{L}$, the fluid velocity seen by the particle can be approximated by a random flow belonging to the Kraichnan ensemble, as discussed in this paper. Then a Hölder exponent $h=1$ or $h<1$ is chosen to study the dissipative or inertial scales of turbulence, respectively.

(ii) For intermediate response times $\tau_{\eta} \ll \tau \ll \tau_{L}$, at least for single or two-particle motions, the fluid velocity seen by the particles can be approximated by an anisotropic generalization of the Kraichnan model [47].

In both asymptotics, the Kraichnan model and its generalization allow for predictions on single- and two-particle properties, many of them were discussed throughout this paper. In the following we discuss them in the context of turbulent suspensions. We focus mostly on two-particle properties at dissipative and inertial scales.

Dissipative range At such small scales, particles form (multi)fractal clusters, which can be quantitatively characterized by the St-dependence of the correlation dimension $\mathcal{D}_{2}$ or, equivalently, of the dimensional deficit $d-\mathcal{D}_{2}$ (in turbulence one can define $\left.\mathrm{St}=\tau / \tau_{\eta}\right)$. Numerical studies [10, 50] show that the qualitative St-dependence of $\mathcal{D}_{2}$ is similar to that observed in the Kraichnan model. Despite such similarities, it is likely that in turbulence, ejection from vortical regions play, at least for small St, an important role [50]. This can clearly not be accounted for in Kraichnan flows, as $\delta$-correlated flows have no persistent structures. The absence of time correlations certainly affects also the scaling behavior when $\mathrm{St} \ll 1$ of the dimension deficit: while in turbulence [5, 40] and timecorrelated stochastic flows [11, 39] it is observed that $d-\mathcal{D}_{2} \propto \mathrm{St}^{2}$, we have shown here that the behavior is linear in St. These discrepancies originate from the fact that white-in-time carrier flows are valid approximations of turbulence only for St $\gg 1$.

Another question concerns the relative dispersion of a particle pair. In the dissipative range, the velocity field is smooth, so that particles separate exponentially with a rate given by the largest Lyapunov exponent $\lambda$. If $\tau \gg \tau_{L}$ the results presented in previous Sections should apply, i.e. $\lambda \propto \mathrm{St}^{-2 / 3}$. For $\tau_{\eta} \ll \tau \ll \tau_{L}$, the anisotropic generalization of the Kraichnan model pre$\operatorname{dicts} \lambda \propto \mathrm{St}^{-5 / 6}$ [47]. However, the measurements of Lyapunov exponents made up to now (see e.g. [27]) do not involve high-enough Stokes and Reynolds numbers to test the validity of these predictions in turbulent flows

Inertial range As shown in this paper, for rough Kraichnan-type carrier flows, particles also form clusters which are however not fractal as they were in the dissipative range. This seems to be in qualitative agreement with the observations made in the inertial range of turbulence: Inhomogeneities have been found in $2 d$ turbulence in the inverse cascade regime [48, 49] as well as in $3 d$ turbulence [50, 51]. However, while in the Kraichnan case the particle distribution depends on the local Stokes number $\operatorname{St}(r)$ only, this does not seem to be the case in turbulence, at least for $\operatorname{St}(r) \ll 1$ as studied in [50] ( which in turbulence is defined by $\operatorname{St}(r)=\tau / \tau_{r}, \tau_{r}$ being the characteristic turbulent time scale associated to the scale $r$ ). In turbulent flows, for small values of $\operatorname{St}(r)$, a different rescaling related to that of the acceleration (and hence pressure) field has been found [50]. However such discrepancies do not question the relevance of the Kraichnan model to turbulent flows as it is expected to be a good approximation only for scales $r$ such that $\tau_{r} \ll \tau$, i.e. $\operatorname{St}(r) \gg 1$. Experiments or direct numerical simulations with high Re and St are thus needed to actually test the validity of the dynamical scaling in terms of $\operatorname{St}(r)$ and to reproduce an equivalent of Fig. 3 for turbulent flows. As far as particle separation is concerned, we have seen in Section IV that at very long times, and thus for separations $r$ such that $\tau \ll \tau_{r}$ one should expect to observe Richardson dispersion. For intermediate times at which the separation is such that $\tau_{\eta} \ll \tau_{r} \ll \tau$, it is predicted in [47] that an intermediate asymptotic regime may emerge with the typical particle separation $r$ growing as $t^{9}$, i.e. much faster than Richardson diffusion. On the numerical and experimental side, we are not aware of any results on the relative dispersion of two heavy particles in the inertial range. Testing the above predictions can be probably done only in experiments where Re can be very high.

In summary, this paper reviews most of current understanding of heavy particle suspensions in Kraichnan-like stochastic flows. In particular, we examined in details two-particle statistics both in smooth and rough velocity fields. Numerical simulations, validated by analytics 
originally derived in this paper, show that particle clustering is more efficient for smooth than rough flows, and can be characterized in terms of the local Stokes number. Detailed predictions can be done in the very small and very large Stokes number asymptotics. In the former we provided an analytical expression for the dimensional deficit for any value of the fluid Hölder exponent. More specifically, it is shown that the departure from a uniform distribution is linear in the Stokes number, a result which is confirmed by numerics. As for the evolution of the relative separation of particle pairs at small separations, a well-verified asymptotic behavior for the Lyapunov exponent is discussed. At larger scales, by converting the scale-dependent Stokes number into a timedependent one, we provided an original way to account for the recovering of tracer-like Richardson diffusion. Finally, the relevance of these results, together with other predictions obtained in recent years from Kraichnan-like models of heavy particle suspensions, to particles in turbulent flows has been discussed.

To conclude this work we suggest two different directions for further investigations. First most of the pre- dictions related to the large-Stokes asymptotics lack numerical or experimental evidence in fluid flows with high Reynolds numbers and particles with huge inertia. Second it is now definitely clear that an important challenge for the near future is to understand whether or not some of the techniques developed for suspensions in random time-uncorrelated flows can be generalized/extended to time-correlated flows. For instance, a quantitative understanding of the small-Stokes-number asymptotics in models that are closer to turbulence would be of great interest to many applications. A first step in this direction has been recently attempted in [52].

\section{Acknowledgments}

We acknowledge useful discussions with S. Musacchio and M. Wilkinson. Part of this work was done while K.T. was visiting Lab. Cassiopée in the framework of the ENS-Landau exchange program.
[1] R.H. Kraichnan, Small-scale structure of a scalar field convected by turbulence, Phys. Fluids 11 (1968) 945-953.

[2] G. Falkovich, K. Gawędzki, and M. Vergassola, Particles and fields in fluid turbulence, Rev. Mod. Phys. 73 (2001) 913-975.

[3] H. Pruppacher and J. Klett, Microphysics of Clouds and Precipitation (Kluwer Academic Publishers,Dordrecht, 1996).

[4] M.B. Pinsky and A.P. Khain, Turbulence effects on droplet growth and size distribution in clouds- a review, J. Aerosol Sci. 28 (1997) 1177-1214.

[5] G. Falkovich, A. Fouxon, and M.G. Stepanov, Acceleration of rain initiation by cloud turbulence, Nature 419 (2002) 151-154.

[6] B.J. Rothschild and T.R. Osborn, Small-scale turbulence and plankton contact rates, J. Plankton Res. 10 (1988) 465-474.

[7] S. Sundby and P. Fossum, Feeding conditions of ArctoNorwegian cod larvae compared with the RothschildOsborn theory on small-scale turbulence and plankton contact rates, J. Plankton Res. 12 (1990) 1153-1162.

[8] J. Mann, S. Ott, H.L. Pécseli and J. Trulsen, Predatorprey encounters in turbulent waters, Phys. Rev. E 65 (2002) 026304.

[9] J.K. Eaton and J.R. Fessler, Preferential concentration of particles by turbulence, Int. J. Multiphase Flow 20 (1994) 169-209.

[10] R.C. Hogan and J.N. Cuzzi, Stokes and Reynolds number dependence of preferential particle concentration in simulated three-dimensional turbulence, Phys. Fluids 13 (2001) 2938-2945.

[11] J. Bec, Fractal clustering of inertial particles in random flows, Phys. Fluids 15 (2003) L81-L84.

[12] G. Falkovich, A. Fouxon, and M. Stepanov, in A. Gyr, W. Kinzelbach (Eds.), Sedimentation and Sediment
Transport, Kluwer Academic Publishers, Dordrecht, 2003, pp. 155-158.

[13] J. Bec, M. Cencini, and R. Hillerbrand, Clustering of heavy particles in random self-similar flow, Phys. Rev. E 75 (2007) 025301.

[14] L.I. Piterbarg, The top Lyapunov exponent for stochastic flow modeling the upper ocean turbulence, SIAM J. Appl. Math. 62 (2002) 777-800.

[15] K. Duncan, B. Mehlig, S. Östlund, and M. Wilkinson, Clustering by mixing flows, Phys. Rev. Lett. 95 (2005) 240602.

[16] J. Bec, M. Cencini, and R. Hillerbrand, Heavy particles in incompressible flows: the large Stokes number asymptotics, Physica D 226 (2007) 11-22.

[17] W.C. Reade and L.R. Collins, Effect of preferential concentration on turbulent collision rates, Phys. Fluids 12 (2000) 2530-2540.

[18] J. Bec, A. Celani, M. Cencini, and S. Musacchio, Clustering and collisions of heavy particles in random smooth flows Phys. Fluids 17 (2005) 073301.

[19] J.-P. Eckmann and D. Ruelle, Ergodic theory of chaos and strange attractors, Rev. Mod. Phys. 57 (1985) 617656.

[20] G. Paladin and A. Vulpiani, Anomalous scaling laws in multifractal objects, Phys. Rep. 156 (1987) 147-225.

[21] L. Arnold, Random Dynamical Systems, Springer Monographs in Mathematics, Berlin/New York, 2003.

[22] T.D. Sauer and J.A. Yorke, Are the dimensions of a set and its image equal under typical smooth functions? Ergodic Theory and Dynamical Systems 17 (1997) 941-956.

[23] L.-P. Wang, A.S. Wexler, and Y. Zhou, On the collision rate of small particles in isotropic turbulence. Part I. Zero-inertia case, Phys. Fluids 10 (1998) 266-276 ; Y. Zhou, L.-P. Wang, and A.S. Wexler, On the collision rate of small particles in isotropic turbulence. Part II. Finite- 
inertia case, Phys. Fluids 10 (1998) 1206-1216.

[24] B. Mehlig and M. Wilkinson, Coagulation by random velocity fields as a Kramers problem, Phys. Rev. Lett. 92 (2004) 250602.

[25] B. Mehlig, M. Wilkinson, K. Duncan, T. Weber, and M. Ljunggren, Aggregation of inertial particles in random flows, Phys. Rev. E 72 (2005) 051104.

[26] M. Wilkinson and B. Mehlig, Caustics in turbulent aerosols, Europhys. Lett. 71 (2005) 186-192.

[27] J. Bec, L. Biferale, G. Boffetta, M. Cencini, S. Musacchio, and F. Toschi, Lyapunov exponents of heavy particles in turbulence, Phys. Fluids 18 (2006) 091702.

[28] K. Gawędzki, and M. Vergassola, Phase transition in the passive scaler advection, Physica D 138 (2000) 63-90.

[29] J.M. Deutsch, Aggregation-disorder transition induced by random forces, J. Phys. A: Math. Gen. 18 (1985) 14491456.

[30] M. Wilkinson and B. Mehlig, Path coalescence transition and its applications, Phys. Rev. E 68 (2003) 040101.

[31] S. Derevyanko, G. Falkovich, K. Turitsyn, and S. Turitsyn, Lagrangian and Eulerian descriptions of inertial particles in random flows, J. Turbulence 8:1 (2007) 1-18.

[32] R. Liboff, Introductory quantum mechanics (AddisonWesley, 2003).

[33] M. Wilkinson, B. Mehlig, S. Östlund, and K.P. Duncan, Unmixing in random flows, preprint nlin.CD/0612061 (2006).

[34] A. Majda, I. Timofeyev, and E. Vanden Eijnden, A mathematical framework for stochastic climate models, Comm. Pure Appl. Math. 54 (2001) 891-974.

[35] S. Nakajima, On quantum theory of transport phenomena: steady diffusion, Progr. Theor. Phys. 20 (1958), 948-959.

[36] R. Zwanzig, Ensemble method in the theory of universality, J. Chem. Phys. 33 (1960) 1338-1341.

[37] H. Risken, The Fokker-Planck Equation, SpringerVerlag, Berlin, 1989.

[38] S. Chaturvedi and F. Shibata, Time-convolutionless operator formalism for elimination of fast variables. Applications to Brownian motion, Zeit. Phys. B 35 (1979) 297-308.

[39] L. I. Zaichik and V. Alipchenkov, Pair dispersion and preferential concentration of particles in isotropic turbu- lence, Phys. Fluids 15 (2003) 1776-1787.

[40] G. Falkovich and A. Pumir, Intermittent distribution of heavy particles in a turbulent flow, Phys. Fluids 16 (2004) L47-L50.

[41] H. Sigurgeirsson and A.M. Stuart, A model for preferential concentration, Phys. Fluids 14 (2002) 4352-4361.

[42] B. Hunt and V. Kaloshin, How projections affect the dimension spectrum of fractal measures, Nonlinearity 10 (1997) 1031-1046.

[43] E. Balkovsky, G. Falkovich and A. Fouxon, Intermittent distribution of inertial particles in turbulent flows, Phys. Rev. Lett. 86 (2001) 2790-2793.

[44] L. Biferale, M. Cencini, A. Lanotte, M. Sbragaglia, and F. Toschi, Anomalous scaling and universality in hydrodynamic systems with power-law forcing, New J. Phys. 6 (2004) 37.

[45] P. Horvai, Lyapunov exponent for inertial particles in the 2D Kraichnan model as a problem of Anderson localization with complex valued potential, preprint nlin.CD/0511023 (2005).

[46] U. Frisch, Turbulence: the legacy of A.N. Kolmogorov, (Cambridge University Press, Cambridge UK, 1995).

[47] I. Fouxon and P. Horvai, Single and two-particle motion of heavy particles in turbulence, preprint arXiv:0704.3893 (2007).

[48] G. Boffetta, F. De Lillo, and A. Gamba, Large scale inhomogeneity of inertial particles in turbulent flow, Phys. Fluids 16 (2004) L20-L23.

[49] L. Chen, S. Goto, and J. C. Vassilicos, Turbulent clustering of stagnation points and inertial particles, J. Fluid Mech. 553 (2006) 143-154.

[50] J. Bec, L. Biferale, M. Cencini, A. Lanotte, S. Musacchio, and F. Toschi, Heavy particle concentration in turbulence at dissipative and inertial scales, Phys. Rev. Lett. 98 (2007) 084502.

[51] H. Yoshimoto and S. Goto, Self-similar clustering of inertial particles in homogeneous turbulence, J. Fluid Mech. 577 (2007) 275-286.

[52] G. Falkovich, S. Musacchio, L. Piterbarg, M. Vucelja, Inertial particles driven by a telegraph noise, Phys. Rev. E 76 (2007) 026313. 